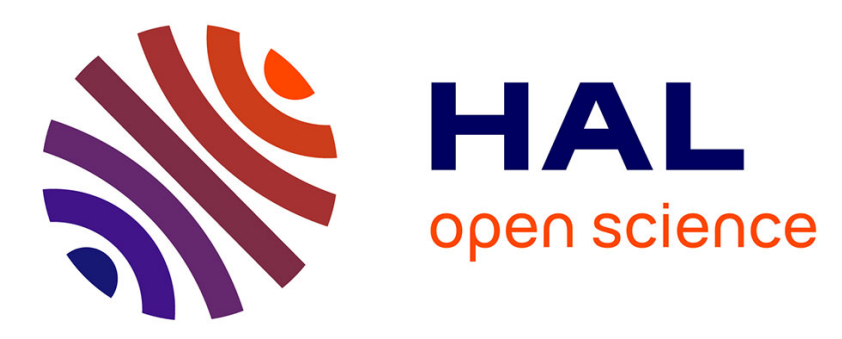

\title{
A conservative Embedded Boundary method for an inviscid compressible flow coupled with a fragmenting structure
}

Maria Adela Puscas, Laurent Monasse, Alexandre Ern, Christian Tenaud, Christian Mariotti

\section{To cite this version:}

Maria Adela Puscas, Laurent Monasse, Alexandre Ern, Christian Tenaud, Christian Mariotti. A conservative Embedded Boundary method for an inviscid compressible flow coupled with a fragmenting structure. International Journal for Numerical Methods in Engineering, 2015, 103 (13), pp.241-262. hal-01071619v2

\section{HAL Id: hal-01071619 \\ https://hal.science/hal-01071619v2}

Submitted on 1 Feb 2015

HAL is a multi-disciplinary open access archive for the deposit and dissemination of scientific research documents, whether they are published or not. The documents may come from teaching and research institutions in France or abroad, or from public or private research centers.
L'archive ouverte pluridisciplinaire HAL, est destinée au dépôt et à la diffusion de documents scientifiques de niveau recherche, publiés ou non, émanant des établissements d'enseignement et de recherche français ou étrangers, des laboratoires publics ou privés. 


\title{
A conservative Embedded Boundary method for an inviscid compressible flow coupled with a fragmenting structure
}

\author{
Maria Adela Puscas ${ }^{1,2,3}$, Laurent Monasse ${ }^{1}$, Alexandre Ern $^{1}$, Christian Tenaud $^{3}$, \\ Christian Mariotti ${ }^{2}$ \\ ${ }^{1}$ Université Paris-Est, CERMICS (ENPC), \\ 77455 Marne la Vallée cedex, France \\ email: \{puscasa, ern, monassel\}@cermics.enpc.fr \\ ${ }^{2}$ CEA-DAM-DIF 91297 Arpajon, France \\ email: $\{$ adela.puscas, christian.mariotti\}@cea.fr \\ ${ }^{3}$ LIMSI-CNRS, 91403 Orsay, France \\ email: $\{$ adela.puscas, christian.tenaud\}@limsi.fr
}

\begin{abstract}
We present an Embedded Boundary method for the interaction between an inviscid compressible flow and a fragmenting structure. The fluid is discretized using a Finite Volume method combining LaxFriedrichs fluxes near the opening fractures, where the density and pressure can be very low, with highorder monotonicity-preserving fluxes elsewhere. The fragmenting structure is discretized using a Discrete Element method based on particles, and fragmentation results from breaking the links between particles. The fluid-solid coupling is achieved by an Embedded Boundary method using a cut-cell Finite Volume method that ensures exact conservation of mass, momentum, and energy in the fluid. A time explicit approach is used for the computation of the energy and momentum transfer between the solid and the fluid. The Embedded Boundary method ensures that the exchange of fluid and solid momentum and energy is balanced. Numerical results are presented for two- and three-dimensional fragmenting structures interacting with shocked flows.
\end{abstract}

Key Words: Fluid-structure interaction, Finite Volume, Embedded Boundary, Conservative method, Fragmenting structure

\section{Introduction}

In the present work, we are interested in the interaction of a shocked fluid with a fragmenting structure. The characteristic time scales of these phenomena are extremely short. The driving effect is the fluid overpressure, and viscous effects play a lesser role in the dynamics of the system, so that we consider an inviscid fluid. An important class of methods for fluid-structure interaction hinges on a partitioned approach, where the fluid and the solid equations are solved separately, and an interface module is used to exchange information between the fluid and the solid solvers. Two main types of methods have been developed in this context: Arbitrary Lagrangian-Eulerian (ALE) methods [9, 19] and fictitious domain methods $[5,11,12,14,15,27,36]$. The ALE method deforms the fluid domain in order to follow the movement of the structure. This method hinges on a mesh fitting the solid boundaries, and this often involves costly remeshing of the fluid domain when the solid goes through large displacements and especially fragmentation. Fictitious domain methods work on a fixed fluid grid. The solid is superimposed to the fluid grid, and additional terms are introduced in the fluid solver to impose the fluid boundary conditions at the solid boundary. Such methods can treat large displacements of the solid and changes in the topology of the fluid domain without remeshing. Various types of fictitious domain methods have been proposed. In particular, Conservative Embedded Boundary methods [3, 13, 17, 18, 26, 28, 29, 31] have been developed for elliptic problems and compressible fluids, so that the spatial discretization conserves mass, momentum, and energy in the fluid.

Coupling fluids with fragmenting structures has already been addressed in the literature. An Embedded Boundary method for a fluid interacting with a fragmenting thin shell was developed in $[2,6]$. The method couples a Lagrangian fragmenting thin shell discretized by a Finite Element method and an Eulerian fluid flow discretized by a Finite Volume method on a Cartesian grid. This method uses a Level 
Set approach to track the immersed solid surface, and a Ghost Fluid method to impose the boundary conditions at the fluid-structure interface. The thin shell fracture criterion is based on a cohesive interface method and uses pre-fractured elements to model the fracture, so that the knowledge of where the fracture will occur is required. An Immersed Particle method [34] was used in [30] for the interaction of a compressible fluid with a fragmenting thin shell, without a priori knowledge of the fracture location. The fracture is modelled by a cracking particle method using a local partition of unity. This method treats both fluid and structure by meshfree particle methods, and the solid is immersed in the fluid. The fluid model is Lagrangian and for very large deformation situations, a re-initialization of the fluid particles is necessary (defaulting energy conservation). Another Embedded Boundary method for the interaction between a compressible flow and a fragmenting thin shell was developed in [20], also without a priori knowledge of the fracture location. The fluid is discretized using a Finite Volume method. The method for the fragmenting structure combines an Extended Finite Element method (X-FEM) [8, 33] with cohesive law and element deletion.

In the present work, we develop an Embedded Boundary method for the three-dimensional interaction between a compressible, inviscid fluid and a three-dimensional fragmenting structure. We extend the approach developed in $[28,29]$ for the coupling with a three-dimensional rigid or deformable structure without fragmentation. Our starting point is a high-order monotonicity-preserving Finite Volume method with directional operator splitting [4] for the Euler equations in conservative form and a Discrete Element method [25] for the structure. This method discretizes the structure using particles, and each particle is governed by the classical equations of mechanics. The particles interact through forces and torques. The Discrete Element method treats naturally fragmentation by breaking links between particles. In the present work, we focus on the feasibility study of the fluid-structure coupling method to deal with fragmentation, so that it is sufficient at this stage to employ a simple model for the breaking criterion based on a maximal relative elongation of particles link. One limitation of the present method is that is does not take into account the possible contact between particles during the ballistic flight after fragmentation.

An important aspect of the present work is to deal with fluid penetration inside the opening gaps when particle links are broken. Considering mode I opening, we observe that the fluid boundary conditions only enforce non-penetration so that the fluid velocity inside the gap can in fact differ from the gap opening velocity. Moreover, the fluid cannot penetrate into the gap faster than a velocity of the order of the speed of sound (as we further elaborate below based on a Riemann problem with vacuum), while the gap can open at a higher speed. The consequence of this modeling is the possible presence of so-called vacuum cells where the fluid pressure and the density are zero or very low. We consider the Lax-Friedrichs numerical flux near these cells in order to avoid division by pressure or density. The Lax-Friedrichs flux is able to compute a stable approximation to the Riemann problem in the presence of vacuum [35]. Away from vacuum cells, the high-order flux from [4] is used. In our numerical experiments, we indeed observe the presence of vacuum cells over relatively short times before the gap is filled with fluid; we also notice that this feature is reproduced independently of the considered grid refinement level.

The coupling algorithm is based on an Embedded Boundary method with an explicit time-marching procedure. The algorithm does not require remeshing and allows fluid to pass through the opening gaps of the structure without any a priori knowledge of where these gaps occur. As in the case without fragmentation, we employ a cut-cell modification of the Finite Volume method, which ensures exact conservation of mass, momentum, and energy quantities in the fluid. Moreover, the exchange of fluid and solid momentum and energy through their interface is exactly balanced up to round-off error in the description of the geometry of cut cells. As the solid time integration scheme is symplectic, it preserves a discrete energy (which is a close approximation of the exact energy). Energy conservation of the coupled discrete system can therefore be verified numerically only, and we show in our test cases that our strategy gives excellent results.

The paper is organized as follows. In Section 2, we set the notation and recall the coupling method without fragmentation. In Section 3, we present the coupling method with fragmentation. In Section 4, we discuss numerical results. Finally, conclusions are drawn in Section 5. 


\section{Coupling without fragmentation}

\subsection{Fluid discretization}

The inviscid compressible flow is modelled by the Euler equations. The equations are written in conservative form expressing conservation of mass, momentum, and energy as follows:

$$
\frac{\partial}{\partial t} U+\frac{\partial}{\partial x} F(U)+\frac{\partial}{\partial y} G(U)+\frac{\partial}{\partial z} H(U)=0
$$

where $U=(\rho, \rho u, \rho v, \rho w, \rho E)^{t}$ with $\rho$ the mass density, $p$ the pressure, $(u, v, w)$ the Cartesian components of the velocity vector $\vec{u}$, and $E$ the total energy. The pressure is modelled by the state equation of a perfect gas: $p=\rho(\gamma-1)\left(E-\frac{1}{2}\left(u^{2}+v^{2}+w^{2}\right)\right), \gamma=1.4$ being the ratio of specific heats, assumed to be constant. The speed of sound in the fluid is $c^{2}=\frac{\gamma p}{\rho}$. The fluid flux functions are given by

$$
F(U)=\left(\begin{array}{c}
\rho u \\
\rho u^{2}+p \\
\rho u v \\
\rho u w \\
(\rho E+p) u
\end{array}\right), G(U)=\left(\begin{array}{c}
\rho v \\
\rho u v \\
\rho v^{2}+p \\
\rho v w \\
(\rho E+p) v
\end{array}\right), H(U)=\left(\begin{array}{c}
\rho w \\
\rho u w \\
\rho v w \\
\rho w^{2}+p \\
(\rho E+p) w
\end{array}\right)
$$

\subsubsection{Fluid grid}

In Embedded Boundary methods, the solid is superimposed to the fluid grid. As a result, some cells of the fluid grid are masked by the solid and are named "solid cells", some others are completely included in the flow domain and are called "fluid cells", and the remaining ones are intersected by the surface of the solid and are referred to as "cut cells" (see Fig. 1). We denote by $\Omega_{\text {Solid }}(t)$ the solid domain and by $\Omega_{\text {Fluid }}(t)$ the fluid domain at time $t$.

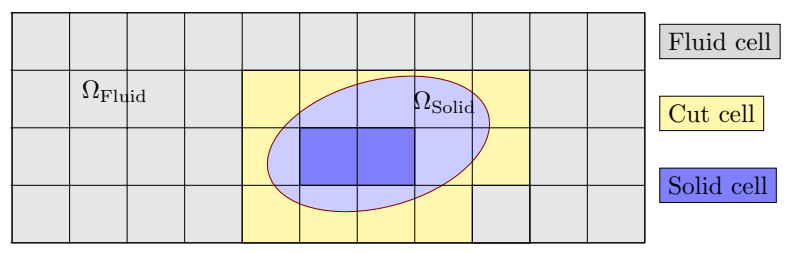

Figure 1: Solid superimposed to the fluid grid

We consider a Cartesian fluid grid, and we denote with integer subscripts $i, j, k$ quantities related to the center of cells and with half-integer subscripts quantities related to the center of faces of cells. For instance, the interface between cells $C_{i, j, k}$ and $C_{i+1, j, k}$ is denoted by $\partial C_{i+\frac{1}{2}, j, k}$. The time step is taken constant for simplicity. We introduce the discrete times $t^{n}=n \Delta t$, for all $n \geq 0$.

The relevant geometric quantities describing the intersection between the moving solid and the cut cell $C_{i, j, k}$ are:

- The volume fraction $0 \leqslant \Lambda_{i, j, k}^{n} \leqslant 1$ occupied by the solid in the cell $C_{i, j, k}$ at time $t^{n}$.

- The side area fractions $0 \leqslant \lambda_{i \pm \frac{1}{2}, j, k}^{n}, \lambda_{i, j \pm \frac{1}{2}, k}^{n}, \lambda_{i, j, k \pm \frac{1}{2}}^{n} \leqslant 1$ of each cell face at time $t^{n}$.

The three-dimensional geometric algorithms used for the detection of the cut cells and the computation of the intersection between the solid and the fluid grid are described in [29].

\subsubsection{Cut cells}

In a cut cell $C_{i, j, k}\left(0<\Lambda_{i, j, k}^{n+1}<1\right)$ of size $\left(\Delta x_{i, j, k}, \Delta y_{i, j, k}, \Delta z_{i, j, k}\right)$, we consider the following approximation of (1):

$$
\left(1-\Lambda_{i, j, k}^{n+1}\right) U_{i, j, k}^{n+1}=\left(1-\Lambda_{i, j, k}^{n+1}\right) U_{i, j, k}^{n}+\Delta t \Phi_{i, j, k, \text { fluid }}^{n}+\Delta t \Phi_{i, j, k, \text { solid }}^{n}+\Delta U_{i, j, k}^{n, n+1},
$$

where $U_{i, j, k}^{n}$ is the numerical approximation of the exact solution over the cell $C_{i, j, k}$ at time $t^{n}$, and the fluid net flux $\Phi_{i, j, k, \text { fluid }}^{n}$ is given by (see [29] for details) 


$$
\begin{aligned}
\Phi_{i, j, k, \text { fluid }}^{n}= & \frac{\left(1-\lambda_{i-\frac{1}{2}, j, k}^{n+1}\right) F_{i-\frac{1}{2}, j, k}^{n}-\left(1-\lambda_{i+\frac{1}{2}, j, k}^{n+1}\right) F_{i+\frac{1}{2}, j, k}^{n}}{\Delta x_{i, j, k}} \\
& +\frac{\left(1-\lambda_{i, j-\frac{1}{2}, k}^{n+1}\right) G_{i, j-\frac{1}{2}, k}^{n}-\left(1-\lambda_{i, j+\frac{1}{2}, k}^{n+1}\right) G_{i, j+\frac{1}{2}, k}^{n}}{\Delta y_{i, j, k}} \\
& +\frac{\left(1-\lambda_{i, j, k-\frac{1}{2}}^{n+1}\right) H_{i, j, k-\frac{1}{2}}^{n}-\left(1-\lambda_{i, j, k+\frac{1}{2}}^{n+1}\right) H_{i, j, k+\frac{1}{2}}^{n}}{\Delta z_{i, j, k}},
\end{aligned}
$$

where $F_{i \pm 1 / 2, j, k}^{n}, G_{i, j \pm 1 / 2, k}^{n}, H_{i, j, k \pm 1 / 2}^{n}$ are numerical fluxes approximating the time-average of the corresponding physical flux over the time interval $\left[t^{n}, t^{n+1}\right]$ and evaluated at $\partial C_{i \pm \frac{1}{2}, j, k}, \partial C_{i, j \pm \frac{1}{2}, k}$, and $\partial C_{i, j, k \pm \frac{1}{2}}$, respectively. We use the unidimensional One-Step Monotonicity-Preserving (OSMP) highorder scheme [4]. As in [28], the extension to the multidimensional case is made with a directional operator splitting [32] consisting in solving alternately the one-dimensional problem in each direction. We denote by $\bar{p}_{x}^{n}, \bar{p}_{y}^{n}$, and $\bar{p}_{z}^{n}$ the pressures used in the resolution of the one-dimensional problems in the $x, y$, and $z$ directions, respectively. The solid flux $\Phi_{i, j, k \text {, solid }}^{n}$ resulting from the presence of the solid boundaries in the cut cell and the swept amount $\Delta U_{i, j, k}^{n, n+1}$ are detailed in Section 2.3. The superscript $n+1$ in $\Lambda^{n+1}$ in the right-hand side of (2) is due to the fact that the whole geometric effect of boundary movement is transferred to the swept amount $\Delta U_{i, j, k}^{n, n+1}$ in order to enable the boundary to change fluid cell during the time step.

The CFL stability condition of the fluid scheme is

$$
\Delta t<\min _{i, j, k}\left(1-\Lambda_{i, j, k}\right)\left(\frac{\Delta x_{i, j, k}}{\left|u_{i, j, k}\right|+c_{i, j, k}}, \frac{\Delta y_{i, j, k}}{\left|v_{i, j, k}\right|+c_{i, j, k}}, \frac{\Delta z_{i, j, k}}{\left|w_{i, j, k}\right|+c_{i, j, k}}\right) .
$$

In small cut-cells where the solid volume fraction is, say, greater than 0.5 , the time step should be decreased to an unacceptably small value. Several approaches are available to ensure stability without a drastic reduction of the time step and at the same time preserve the conservation properties of the scheme. For example, [18] proposes a conservative mixing of the cut cell with a fluid cell, found in the direction of the outward normal to the solid boundary present in the cut cell. This treatment possibly affects the order of the method in the vicinity of the solid boundary, but does not impact the conservation properties. The accuracy could be enhanced by taking into account the effective shape of the cut cells in the fluid flux computation, which is not done in this work.

\subsubsection{Solid cells}

The stencil used in the OSMP flux function can overlap with the solid. Near the solid, the states needed to calculate the fluid fluxes may be located in cells completely occupied by the solid (solid cells, $\Lambda=1$ ). In this situation, we define in these solid cells a fictitious state from the states associated with the mirror cells relatively to the fluid-solid interface (see [29]).

\subsection{Solid discretization}

The deformable moving solid is discretized by the Discrete Element method using a finite number of rigid particles (Fig. 2). Each particle is governed by the classical equations of mechanics. The particles interact through forces and torques. The expression of these forces and torques allows one to recover the macroscopic behavior of the solid $[22,25]$.

\subsubsection{Forces and torques between particles}

Various quantities are attached to a generic solid particle $I$, namely the mass $m_{I}$, the volume $V_{I}$ and the free volume $V_{I}^{l}$, the position of the center of mass $\vec{X}_{I}$, the velocity of the center of mass $\vec{V}_{I}$, the rotation matrix $\mathbf{Q}_{I}$, the angular momentum matrix $\mathbf{P}_{I}$, and the principal moments of inertia $I_{I}^{i}$, $i \in\{1,2,3\}$. Let $\mathbf{D}_{I}=\operatorname{diag}\left(d_{I}^{1}, d_{I}^{2}, d_{I}^{3}\right)$ with $d_{I}^{i}=\frac{1}{2}\left(I_{I}^{1}+I_{I}^{2}+I_{I}^{3}\right)-I_{I}^{i}, i \in\{1,2,3\}$. We denote by $\tau_{I}$ the neighboring particles linked to particle $I$. Various quantities are attached to the link between the particle 
$I$ and a neighboring particle $J \in \tau_{I}$, namely the distance between these particles $D_{I J}$, the contact surface $S_{I J}$, the center of mass of the contact surface $\vec{G}_{I J}$, the exterior normal vector at the contact surface $\vec{n}_{I J}$, and the principal moments of the contact surface $I_{I J}^{s}$ and $I_{I J}^{t}$. We also define two orthogonal vectors at the contact surface $\vec{s}_{I J}$ and $\vec{t}_{I J}$ forming an orthonormal basis with $\vec{n}_{I J} \cdot \vec{X}_{I}^{0}, D_{I J}^{0}$, and $\vec{n}_{I J}^{0}$ denote the initial values for $\vec{X}_{I}, D_{I J}$, and $\vec{n}_{I J}$ respectively.

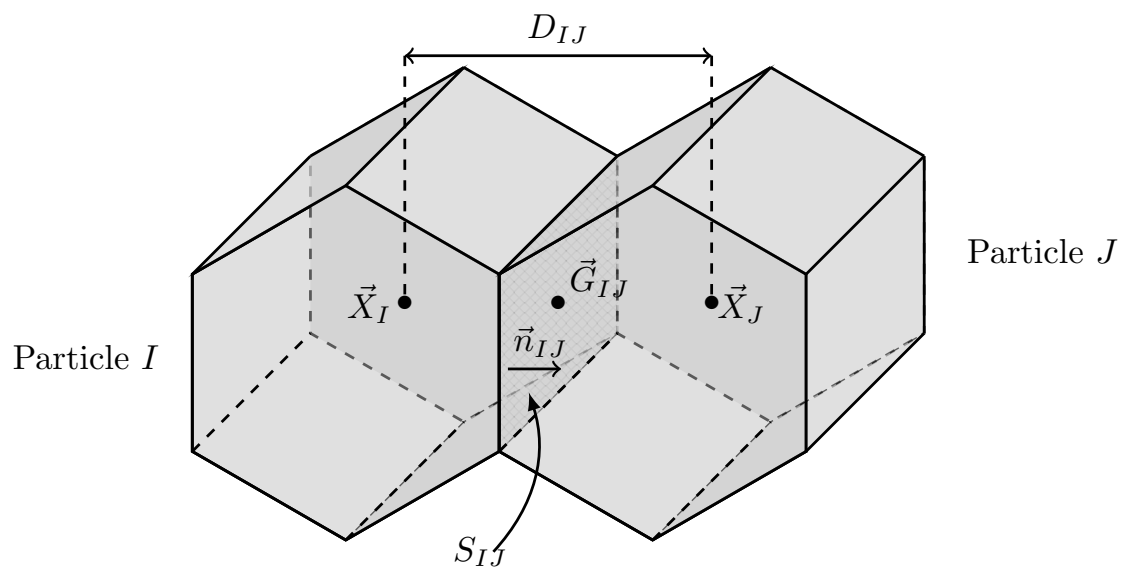

Figure 2: Solid discretization

Forces and torques between particles $I$ and $J$ are modelled by a linear elasticity behavior [22, 25]. The solid is characterized by the Young modulus $E$ and by the Poisson ratio $\nu$. The forces and torques between particles are derived from an Hamiltonian formulation. We briefly recall the expression of these forces and torques, for a detailed review see [22, 25]. The force between particles $I$ and $J \in \tau_{I}$ is given by

$$
\vec{F}_{I J}=\frac{S_{I J}}{D_{I J}^{0}} \frac{E}{1+\nu} \overrightarrow{\Delta u_{I J}}+S_{I J} \frac{E \nu}{(1+\nu)(1-2 \nu)} \varepsilon_{I J}^{v}\left(\vec{n}_{I J}+\frac{1}{D_{I J}} \overrightarrow{\Delta u_{I J}}-\frac{1}{D_{I J}}\left(\overrightarrow{\Delta u_{I J}} \cdot \vec{n}_{I J}\right) \vec{n}_{I J}\right),
$$

where $\overrightarrow{\Delta u_{I J}}=\vec{X}_{I}-\vec{X}_{J}+\mathbf{Q}_{J} \cdot \vec{X}_{J}^{0} \vec{G}_{I J}-\mathbf{Q}_{I} \cdot \vec{X}_{I}^{0} \vec{G}_{I J}$ is the difference of the displacement vectors of interface point $\vec{G}_{I J}$ driven by particles $I$ and $J$, and the volumetric deformation of the link between $I$ and $J, \varepsilon_{I J}^{v}=\varepsilon_{I}^{v}+\varepsilon_{J}^{v}$, is the sum of the volumetric deformation of $I$ and $J$, where

$$
\varepsilon_{I}^{v}=\sum_{J \in \tau_{I}} \frac{1}{2} \frac{S_{I J}}{V_{I}+3 \frac{\nu}{1-2 \nu} V_{I}^{l}} \overrightarrow{\Delta u_{I J}} \cdot \vec{n}_{I J}
$$

The torque between particles $I$ and $J$ is given by $\overrightarrow{\mathcal{M}}_{I J}=\overrightarrow{\mathcal{M}}_{I J}^{t}+\overrightarrow{\mathcal{M}}_{I J}^{f}$, where $\overrightarrow{\mathcal{M}}_{I J}^{t}$ denotes the torque of force $\vec{F}_{I J}$ with respect to the center of gravity of the interface $\vec{G}_{I J}$ :

$$
\overrightarrow{\mathcal{M}}_{I J}^{t}=\frac{S_{I J}}{D_{I J}^{0}} \frac{E}{1+\nu}\left(\mathbf{Q}_{I} \cdot \vec{X}_{I}^{0} \vec{G}_{I J}\right) \wedge \overrightarrow{\Delta u}_{I J}+S_{I J} \frac{E \nu}{(1+\nu)(1-2 \nu)} \varepsilon_{I J}^{v}\left(\mathbf{Q}_{I} \cdot \vec{X}_{I}^{0} \vec{G}_{I J}\right) \wedge \vec{n}_{I J}
$$

and $\overrightarrow{\mathcal{M}}_{I J}^{f}$ denotes the flexion-torsion torque:

$$
\overrightarrow{\mathcal{M}}_{I J}^{f}=\frac{S_{I J}}{D_{I J}^{0}}\left(\alpha_{n}\left(\mathbf{Q}_{I} \cdot \vec{n}_{I J}^{0}\right) \wedge\left(\mathbf{Q}_{J} \cdot \vec{n}_{I J}^{0}\right)+\alpha_{s}\left(\mathbf{Q}_{I} \cdot \vec{s}_{I J}\right) \wedge\left(\mathbf{Q}_{J} \cdot \vec{s}_{I J}\right)+\alpha_{t}\left(\mathbf{Q}_{I} \cdot \vec{t}_{I J}\right) \wedge\left(\mathbf{Q}_{J} \cdot \vec{t}_{I J}\right)\right) .
$$

The coefficients $\alpha_{n}, \alpha_{s}$, and $\alpha_{t}$ are chosen to recover the exact flexion and torsion of a beam:

$\alpha_{n}=\frac{(1+2 \nu) E}{4(1+\nu) S_{I J}}\left(I_{I J}^{s}+I_{I J}^{t}\right), \alpha_{s}=\frac{E}{4(1+\nu) S_{I J}}\left((3+2 \nu) I_{I J}^{s}-(1+2 \nu) I_{I J}^{t}\right)$, and $\alpha_{t}=\frac{E}{4(1+\nu) S_{I J}}((3+$ $\left.2 \nu) I_{I J}^{t}-(1+2 \nu) I_{I J}^{s}\right)$.

\subsubsection{Time integration scheme}

The explicit time-integration scheme for the solid consists of the Verlet scheme for translation and the RATTLE scheme for rotation. For particle $I$, it takes the following form: 


$$
\begin{aligned}
\vec{V}_{I}^{n+\frac{1}{2}} & =\vec{V}_{I}^{n}+\frac{\Delta t}{2 m_{I}}\left(\vec{F}_{I, \text { int }}^{n}+\vec{F}_{I, \text { fluid }}^{n}\right), \\
\vec{X}_{I}^{n+1} & =\vec{X}_{I}^{n}+\Delta t \vec{V}_{I}^{n+\frac{1}{2}}, \\
\mathbf{P}_{I}^{n+\frac{1}{2}} & =\mathbf{P}_{I}^{n}+\frac{\Delta t}{4} \mathbf{j}\left(\overrightarrow{\mathcal{M}}_{I, \text { int }}^{n}+\overrightarrow{\mathcal{M}}_{I, \text { fluid }}^{n}\right) \mathbf{Q}_{I}^{n}+\frac{\Delta t}{2} \mathbf{\Upsilon}_{I}^{n} \mathbf{Q}_{I}^{n}, \\
\mathbf{Q}_{I}^{n+1} & =\mathbf{Q}_{I}^{n}+\Delta t \mathbf{P}_{I}^{n+\frac{1}{2}} \mathbf{D}_{I}^{-1}, \\
\vec{V}_{I}^{n+1} & =\vec{V}_{I}^{n+\frac{1}{2}}+\frac{\Delta t}{2 m_{I}}\left(\vec{F}_{I, \text { int }}^{n+1}+\vec{F}_{I, \text { fluid }}^{n}\right), \\
\mathbf{P}_{I}^{n+1} & =\mathbf{P}_{I}^{n+\frac{1}{2}}+\frac{\Delta t}{4} \mathbf{j}\left(\overrightarrow{\mathcal{M}}_{I, \text { int }}^{n+1}+\overrightarrow{\mathcal{M}}_{I, \text { fluid }}^{n}\right) \mathbf{Q}_{I}^{n+1}+\frac{\Delta t}{2} \tilde{\mathbf{\Upsilon}}_{I}^{n+1} \mathbf{Q}_{I}^{n+1},
\end{aligned}
$$

where in (6), $\mathbf{\Upsilon}_{I}^{n}$ is a symmetric matrix such that $\left(\mathbf{Q}_{I}^{n+1}\right)^{t} \mathbf{Q}_{I}^{n+1}=\mathbf{I}$, with $\mathbf{I}$ the identity matrix in $\mathbb{R}^{3}$, and in (9), $\tilde{\mathbf{\Upsilon}}_{I}^{n+1}$ is a symmetric matrix such that $\left(\mathbf{Q}_{I}^{n+1}\right)^{t} \mathbf{P}_{I}^{n+1} \mathbf{D}_{I}^{-1}+\mathbf{D}_{I}^{-1}\left(\mathbf{P}_{I}^{n+1}\right)^{t} \mathbf{Q}_{I}^{n+1}=\mathbf{0}$. The map $\mathbf{j}: \mathbb{R}^{3} \rightarrow \mathbb{R}^{3 \times 3}$ is such that $\mathbf{j}(\vec{x}) \vec{y}=\vec{x} \wedge \vec{y}$ for all $\vec{x}, \vec{y} \in \mathbb{R}^{3}$. The force $\vec{F}_{I, \text { int }}^{n}$ and the torque $\overrightarrow{\mathcal{M}}_{I, \text { int }}^{n}$ result from the interaction of particle $I$ with its neighboring particles: $\vec{F}_{I, \text { int }}^{n}=\sum_{J \in \tau_{I}} \vec{F}_{I J}^{n}$ and $\overrightarrow{\mathcal{M}}_{I, \text { int }}^{n}=\sum_{J \in \tau_{I}} \overrightarrow{\mathcal{M}}_{I J}^{n}$. The forces $\vec{F}_{I, \text { fluid }}^{n}$ and $\overrightarrow{\mathcal{M}}_{I, \text { fluid }}^{n}$ are respectively the fluid force and torque applied to the particle $I$, and are detailed in Section 2.3.

\subsubsection{Time step constraint}

The time-integration scheme for the solid being explicit, the time step is restricted by a CFL stability condition. This condition states that the displacement of each solid particle $I$ during one time step should be less than the characteristic size of the particles, and the rotation during one time step should be less than $\frac{\pi}{8}$, see [25]. In the case of coupling with fluid, an additional condition applies to the displacement of the solid, requiring it to be less than one fluid grid cell size in the course of the time step, so that the solid boundary crosses at most one fluid grid cell per time step.

\subsubsection{Definition of fluid-solid interface}

The particles have a polyhedral shape and are assumed to be star-shaped with respect to their center of mass, and their faces are assumed to be star-shaped with respect to their center of mass. We define the thickness of a solid particle as the radius of its largest inscribed sphere. We assume that the solid particles have a thickness larger than or equal to two fluid grid cells. The faces of the solid particles in contact with the fluid are collected in the set $\mathfrak{F}$. A generic element of $\mathfrak{F}$ is denoted by $\mathcal{F}$ and is called a wet solid face. The fluid-solid interface consists of all the wet solid faces. Owing to the movement of the solid, the wet solid faces are time-dependent sets in $\mathbb{R}^{3}$, and we set $\mathcal{F}^{n}=\mathcal{F}\left(t^{n}\right)$ for all $n \geq 0$. For each wet solid face $\mathcal{F}(t)$, we consider its surface $A_{\mathcal{F}}(t)$ and its normal $\vec{\nu}_{\mathcal{F}}(t)$ (pointing from the solid to the fluid). As long as the solid deforms without fragmentation (see Fig. 3) a continuous interface around the particle assembly is reconstructed as follows. A vertex $a_{i}$ of the initial Discrete Element lattice belongs to one or more polyhedral particles. We define the mean vertex $\bar{a}_{i}$ corresponding to $a_{i}$ as the average of the positions of vertex $a_{i}$ under the rigid body motion of each particle to which this vertex belongs:

$$
\bar{a}_{i}^{n}=\frac{1}{\# \mathcal{P}_{a_{i}}} \sum_{J \in \mathcal{P}_{a_{i}}}\left(X_{J}^{n}+\mathbf{Q}_{J}^{n} \cdot\left(a_{i}^{0}-X_{J}^{0}\right)\right),
$$

where $a_{i}^{0}$ is the initial position of $a_{i}, \mathcal{P}_{a_{i}}$ is the set of particles which share the vertex $a_{i}$, and $\# \mathcal{P}_{a_{i}}$ is the cardinality of the set $\mathcal{P}_{a_{i}}$. Since the mean vertices $\bar{a}_{i}^{n}$ do not remain coplanar in general, the reconstructed fluid-solid interface is the set of triangles supported by the center of mass of the polyhedral particle face and the mean vertices $\bar{a}_{i}^{n}$. The detailed procedure of the boundary reconstruction is described in $[28]$.

\subsection{Fluid-solid time integration}

The computational cost of the fluid and solid methods lies mainly in the evaluation of fluxes on the fluid side and of forces and torques on the solid side. Both methods being time-explicit, we use a coupling 


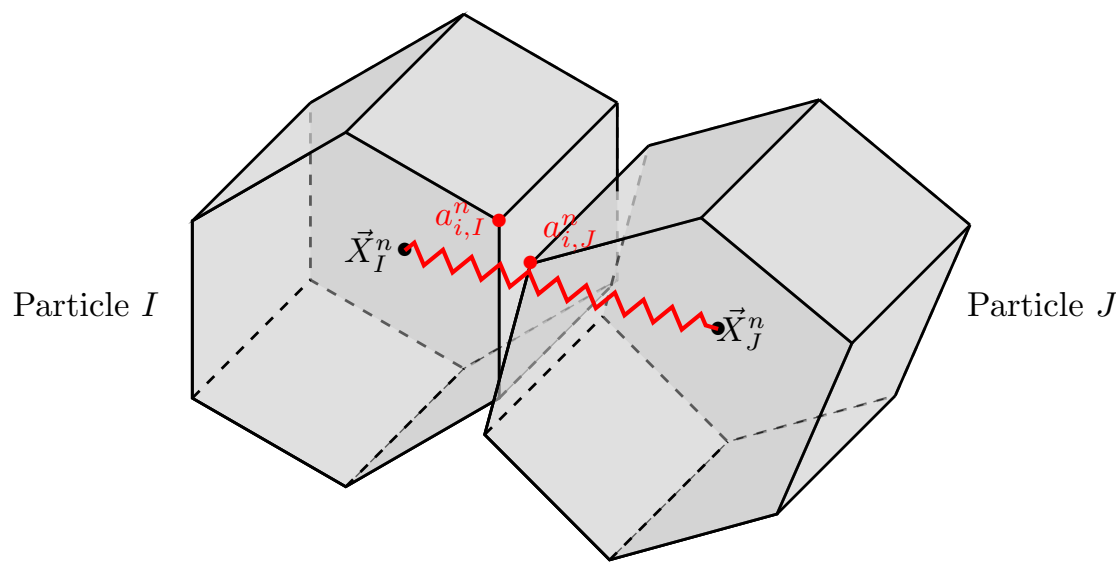

Figure 3: Solid deformation without fragmentation (I and J are still linked)

algorithm based on an explicit time-marching procedure. This is reasonable since, in particular, added mass effects are expected to play a minimal role in the present situations. At the beginning of the time step from $t^{n}$ to $t^{n+1}$, we know the state of the fluid $U^{n}$, the position and rotation of the solid particles $\left(\vec{X}_{I}^{n}, \mathbf{Q}_{I}^{n}\right)$, as well as the velocity of their center of mass and their angular momentum $\left(\vec{V}_{I}^{n}, \mathbf{P}_{I}^{n}\right)$. For the fluid, we need to compute, for each fluid grid cell $C_{i, j, k}$, the fluxes $F_{i \pm 1 / 2, j, k}^{n+\frac{1}{2}}, G_{i, j \pm 1 / 2, k}^{n+\frac{1}{2}}, H_{i, j, k \pm 1 / 2}^{n+\frac{1}{2}}$, the volume fractions $\Lambda_{i, j, k}^{n+1}$, the side area fractions $\lambda_{i \pm 1 / 2, j, k}^{n+1}, \lambda_{i, j \pm 1 / 2, k}^{n+1}, \lambda_{i, j, k \pm 1 / 2}^{n+1}$, the solid fluxes $\Phi_{i, j, k \text {, solid }}^{n}$, and the swept amount $\Delta U_{i, j, k}^{n, n+1}$. For the solid, we need to compute, for each solid particle $I$, the fluid forces $\vec{F}_{I, \text { fluid }}^{n+1}$ and the fluid torques $\overrightarrow{\mathcal{M}}_{I, \text { fluid }}^{n+1}$.

The solid flux for a fluid cut cell $C_{i, j, k}$ is given by

$$
\Phi_{i, j, k, \text { solid }}^{n}=\frac{1}{V_{i, j, k}} \sum_{\left\{\mathcal{F}^{n} \in \mathfrak{F} \mid \mathcal{F}^{n+1} \cap C_{i, j, k} \neq \emptyset\right\}} \phi_{i, j, k, \mathcal{F}}^{n},
$$

where $V_{i, j, k}$ is the volume of $C_{i, j, k}$, and $\phi_{i, j, k, \mathcal{F}}^{n}$ is the solid flux attached to the wet solid face $\mathcal{F}^{n}$ intersecting the cell $C_{i, j, k}$ at time $t^{n+1}$ (as indicated by the notation $\mathcal{F}^{n+1} \cap C_{i, j, k} \neq \emptyset$ ). To facilitate the computation of the solid flux and of the swept amount, we subdivide each solid face $\mathcal{F}$ into a set of triangles, called sub-faces and generically denoted by $f$ (so that $\mathcal{F}=\cup f$ ), that are contained in one fluid grid cell (not necessary the same) at times $t^{n}$ and $t^{n+1}$. We set $f^{n}=f\left(t^{n}\right)$ for all $n \geq 0$. The solid flux attached to the face $\mathcal{F}^{n}$ is then decomposed as

$$
\phi_{i, j, k, \mathcal{F}}^{n}=\sum_{\left\{f^{n} \in \mathcal{F}^{n} \mid f^{n} \subset C_{i, j, k}\right\}}\left(0, \Pi_{x, f}^{n}, \Pi_{y, f}^{n}, \Pi_{z, f}^{n}, \vec{V}_{f}^{n+\frac{1}{2}} \cdot \vec{\Pi}_{f}^{n}\right)^{t},
$$

where

$$
\vec{\Pi}_{f}^{n}=\left(\int_{f^{n}} \bar{p}_{x}^{n} \nu_{x, f}^{n}, \int_{f^{n}} \bar{p}_{y}^{n} \nu_{y, f}^{n}, \int_{f^{n}} \bar{p}_{z}^{n} \nu_{z, f}^{n}\right)^{t},
$$

and $\vec{V}_{f}^{n+\frac{1}{2}}=\vec{V}^{n+\frac{1}{2}}+\vec{\Omega}^{n+\frac{1}{2}} \wedge\left(\vec{X}_{f}^{n}-\vec{X}^{n}\right)$ is the velocity of the center of mass of $f^{n}, \vec{X}_{f}^{n}$ is the center of mass of $f^{n}$, while $\vec{V}^{n+\frac{1}{2}}$ and $\vec{\Omega}^{n+\frac{1}{2}}$ are, respectively, the average velocity and rotation velocity of the solid particle containing $f$. Furthermore, the swept amount is given by

$$
\Delta U_{i, j, k}^{n, n+1}=\sum_{\left\{\mathcal{F}^{n} \in \mathfrak{F} \mid \mathcal{F}^{n+1} \cap C_{i, j, k} \neq \emptyset\right\}} \Delta U_{i, j, k, \mathcal{F}}^{n, n+1},
$$

where the term $\Delta U_{i, j, k, \mathcal{F}}^{n, n+1}$ denotes the amount of $U$ swept by the movement of the wet solid face $\mathcal{F}$ during the time step from $t^{n}$ to $t^{n+1}$. We define a piecewise affine map $\Psi_{n, n+1}$ from $\mathcal{F}^{n}$ to $\mathcal{F}^{n+1}$, preserving the triangular subdivision of $\mathcal{F}$, so that each sub-face satisfies $f^{n}=\Psi_{n, n+1}\left(f^{n+1}\right)$. Then, the swept amount $\Delta U_{i, j, k, \mathcal{F}}^{n, n+1}$ is given by

$$
\Delta U_{i, j, k, \mathcal{F}}^{n, n+1}=\frac{1}{V_{i, j, k}} \sum_{\left\{f^{n+1} \in \mathcal{F}^{n+1} \mid f^{n+1} \subset C_{i, j, k}\right\}} \sum_{\left\{C_{p, q, r} \mid K_{f} \cap C_{p, q, r} \neq \emptyset\right\}} \mathcal{V}_{p, q, r} U_{p, q, r}^{n},
$$


where $\mathcal{V}_{p, q, r}$ is the signed volume of the intersection between the prism $K_{f}$ (whose bases are $f^{n}$ and $f^{n+1}$ ) and the fluid grid cell $C_{p, q, r}$. The detailed procedure to compute the above quantities is described in [29], see also [24].

The fluid force acting on the solid particle $I$ is given by

$$
\vec{F}_{I, \text { fluid }}^{n}=\sum_{\mathcal{F} \in \mathfrak{F}_{I}} \vec{F}_{\mathcal{F}, \text { fluid }}^{n}=-\sum_{\mathcal{F} \in \widetilde{F}_{I}} \vec{\Pi}_{\mathcal{F}}^{n}
$$

where $\mathfrak{F}_{I}$ collects the wet faces of the particle $I$. Similarly, the fluid torque $\overrightarrow{\mathcal{M}}_{I, \text { fluid }}^{n}$ is given by

$$
\overrightarrow{\mathcal{M}}_{I, \text { fluid }}^{n}=\sum_{\mathcal{F} \in \widetilde{F}_{I}} \vec{F}_{\mathcal{F}, \text { fluid }}^{n} \wedge\left(\vec{X}_{\mathcal{F}}^{n}-\vec{X}_{I}^{n}\right) .
$$

Explicit and semi-implicit time integration schemes for the fluid-solid coupling are described in [28, 29].

\section{Coupling with fragmenting structure}

\subsection{Solid fragmentation in the Discrete Element method}

The Discrete Element method deals with solid fragmentation by breaking the link between particles (Fig. 4). The fracture propagates element by element using a fracture criterion defined at the contact faces between particles. Dynamic fragmentation using the Discrete Element method has been studied in [23], using both the discrete Camacho-Ortiz criterion [1] and the continuous Denoual et al. criterion [7]. The Camacho-Ortiz criterion expresses damage as a function of crack opening. When the local stress reaches a threshold, it decreases linearly with the crack opening until the fracture is open. The Denoual et al. criterion is a probabilistic criterion where the damage is introduced per unit volume using a Weibull probability distribution. The coupling method is independent of the breaking criterion used in the solid solver in order to break the link between particles. In the present work, we focus on the feasibility study of the coupling method to deal with fragmentation and we choose a simple breaking criterion, namely the relative elongation at break of the structure, which measures the ability of the material to elongate before rupture under load. The interaction behavior law between the particles in term of forces and torques is still taken here linear elastic in order to simplify the presentation and to verify the conservation of energy by the coupling system. More complex laws between particles can be used by integrating them in the expression of internal forces and torques between particles.

The relative elongation at break is a dimensionless quantity which is a characteristic of the material and which is determined by a tensile test. In the case of a brittle material, the rupture occurs at the end of the elastic domain. The fracture surface is generally perpendicular to the axis of traction. The elongation at break is very low, for instance, for a cast iron it is in the range $(0.3,0.8)$, so that a $1 \mathrm{~m}$ long beam cracks before its elongation reaches $8 \mathrm{~mm}$. For two neighboring particles $I$ and $J$, we compute the relative elongation of the $I-J$ link as follows:

$$
A_{I J} \%=100 \frac{\left\|\vec{X}_{I}^{n}-\vec{X}_{J}^{n}\right\|-\left\|\vec{X}_{I}^{0}-\vec{X}_{J}^{0}\right\|}{\left\|\vec{X}_{I}^{0}-\vec{X}_{J}^{0}\right\|} .
$$

The link is broken when $A_{I J} \%$ exceeds the relative elongation at break.

The swept amount and the boundary reconstruction are computed for every solid face, including the internal faces for which this treatment is not necessary (since contributions from solid vis-a-vis faces cancel). This allows for an effective management of new wet faces when fracture occurs as all faces follow the same treatment. An alternative approach could be to perform these treatments only for the internal faces for which there is a potential risk of fracture (when the elongation at break is close to a critical level).

\section{$3.2 \quad$ Vacuum cells}

During the process of fragmentation, vacuum between solid particles can occur due to the fact that the velocity of the crack propagation can be larger than the penetration velocity of the fluid into the 


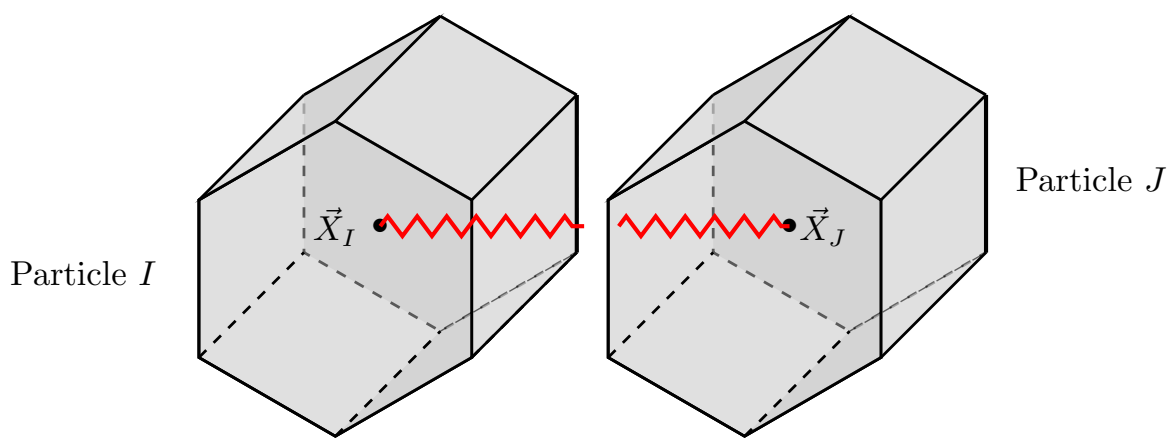

Figure 4: Broken link between particles

opening gap between the particles. The opening gap and fluid penetration velocities can differ since the non-penetration boundary condition only enforces the velocity component normal to the gap lips. Consider for instance the situation presented in Fig. 5. In the case of the Griffith fracture model [16] for a linear elastic material, a theoretical analysis shows that the limiting fracture speed for mode I fracture is the Rayleigh wave speed, which can be estimated by the following expression [10]:

$$
c_{R}=c_{s} \frac{0.862+1.14 \nu}{1+\nu},
$$

where $\nu$ is the Poisson ratio. The compression wave speed $c_{p}$ (a wave in which the disturbance is a compression of the medium) and the shear wave speed $c_{s}$ (a wave in which the disturbance is an elastic deformation perpendicular to the direction of motion of the wave) are given by the following formulas:

$$
c_{p}=\sqrt{\frac{E(1-\nu)}{\rho(1+\nu)(1-2 \nu)}}, \quad c_{s}=\sqrt{\frac{E}{2 \rho(1+\nu)}},
$$

where $E$ is the Young modulus. For mode II and mode III, limiting speeds are the compression and shear wave speed, respectively [10].

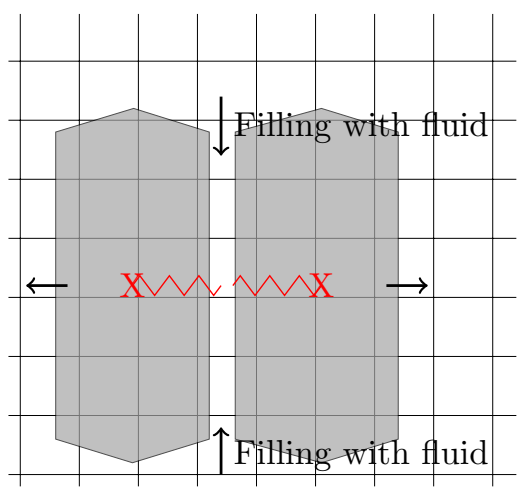

Figure 5: Fluid penetration into the crack due to the broken link between two solid particles

On the fluid part, let us consider the one-dimensional case of a fluid occupying the region $x<0$ and a vacuum region in $x>0$. This leads to the following Riemann problem:

$$
U(x, 0)= \begin{cases}U_{\text {fluid }}, & \text { if } \quad x<0, \\ U_{\text {vacuum }}, & \text { if } \quad x>0,\end{cases}
$$

where $U_{\text {fluid }}$ and $U_{\text {vacuum }}$ are the states in the fluid and vacuum regions, respectively. As in [35], we consider that $U_{\text {vacuum }}=\left(0, u_{0}, 0\right)$, where $u_{0}$ is the velocity of the interface between the two regions (due to the gradient of pressure) given by $u_{0}=u_{\text {fluid }}+\frac{2 c_{\text {fluid }}}{\gamma-1}$. 
In the case of steel $\left(E=2.1 \times 10^{11} \mathrm{~Pa}, \nu=0.3, \rho=7.8 \times 10^{3} \mathrm{~kg} \cdot \mathrm{m}^{-3}\right)$, the estimation of $c_{R}$ is around $3000 \mathrm{~m} \cdot \mathrm{s}^{-1}$. For air at rest under normal atmospheric conditions $\left(c_{\text {fluid }}=340 \mathrm{~m} \cdot \mathrm{s}^{-1}, \gamma=1.4\right)$, the estimation of $u_{0}$ is $1700 \mathrm{~m} \cdot \mathrm{s}^{-1}$. If we consider the crack opening independently from the fluid in mode I, the crack opens at a depth $c_{R} T$ at time $T$. Since the fluid cannot fill the gap faster than $u_{0} T<c_{R} T$, vacuum should occur inside the opening gap.

The vacuum presence leads to fluid cells where the fluid pressure and the density are close to zero. Vacuum also occurs in the case of an internal fracture in the solid with no outlet in the fluid domain. In these cases, the solid cells $(\Lambda=1)$ can become cut cells or fluid cells $(0 \leq \Lambda<1)$ because there is a gap between particles, and the fluid occupying this gap has a very low pressure and density. This leads us to consider a new type of cell, the "vacuum cell", which is characterized by $\Lambda \leq 1$ and a fluid state with vanishing density and pressure.

Herein, we treat the interface between a vacuum cell and a fluid cell by solving the fluid/vacuum Riemann problem (15), $U_{\text {fluid }}$ being the state of the fluid cell in contact with vacuum. The Riemann problem is solved numerically using the Lax-Friedrichs flux (LF), given by

$$
F_{i+1 / 2, j, k}^{n}=\frac{1}{2}\left(F_{i, j, k}^{n}+F_{i+1, j, k}^{n}\right)-\frac{\Delta x_{i, j, k}}{2 \Delta t}\left(U_{i+1, j, k}^{n}-U_{i, j, k}^{n}\right) .
$$

We have chosen this flux because it does not involve any division by pressure or density. It is known that this flux is dissipative [21]. The use of the Lax-Friedrichs flux is limited around the crack region. Once a vacuum cell is filled with fluid, it is no longer a vacuum cell, and the usual OSMP flux is used in the subsequent time steps.

\subsection{Mixing of small cut-cells}

Several solid boundaries may be present in a cut cell after the fragmentation of the solid. Let us consider the situation illustrated in Fig. 5. Two vis-à-vis solid particles are present in one small cut-cell. In the direction of the outward normal to the solid boundary the cells are entirely occupied by the solid, and the neighboring cells are also small cut-cells. Therefore, the usual mixing of small cut-cells cannot be employed. To deal with this situation, we look for a target cell using a recursive algorithm. If the neighboring cells are all either solid cells or small cut-cells, we choose the neighboring cut cell with the largest face aperture as temporary target cell. We iterate until we find a fluid cell or come into a cycle (in this case the target cell is the last cell found before cycling). This ensures that there exists a target cell as well as a fluid path from the original small cut-cell to the target cell.

Let $C_{\text {target }}$ be a fluid cell that has been found as a target cell and $U_{\text {target }}$ the fluid state in that cell. Let $\mathcal{C}_{i, j, k}$ collect all the small cut-cells having $C_{\text {target }}$ as target cell. Defining

$$
U_{\text {Mix }}=U_{\text {target }}+\sum_{C_{i, j, k} \in \mathcal{C}_{i, j, k}}\left(1-\Lambda_{i, j, k}\right) U_{i, j, k}, \quad \text { and } \quad V_{\text {Mix }}=1+\sum_{C_{i, j, k} \in \mathcal{C}_{i, j, k}}\left(1-\Lambda_{i, j, k}\right),
$$

then the new state in $C_{\text {target }}$ is $U_{\text {target }}=\frac{U_{\text {Mix }}}{V_{\text {Mix }}}$. The new state for all $C_{i, j, k} \in \mathcal{C}_{i, j, k}$ is $U_{\text {target }}$.

The mixing procedure is conservative and ensures that the equivalent volume of a small cut-cell is compatible with the CFL condition using the standard-size cells.

\subsection{Time-explicit coupling scheme}

The general procedure for the conservative coupling method can be described by the following steps:

- The fluid fluxes used in (3) are precomputed at all the cell faces of the fluid grid, without taking into account the presence of the solid using the LF flux in regions adjacent to vacuum and the OSMP flux elsewhere.

- The solid internal forces and torques are computed based on the position of the solid particles.

- The fluid pressure forces and torques exerted on the solid are evaluated using (13) and (14).

- The solid is advanced in time.

- The volume fractions and side area fractions are computed using the new position of the fluid-solid interface. The fluid fluxes in (3) are modified using these volume fractions and side area fractions. At this stage, the swept amount is also calculated using (12).

- The solid flux is computed using (11), and the final value of the fluid state is calculated using (2). 
- The small cut-cells are mixed and the solid cells are filled in order to prepare for the next time step.

- The vacuum cells are updated.

The fluid and the solid should advance with the same time step. Thus, we use in the fluid and in the solid solvers the minimum between the fluid and the solid time steps provided by the respective CFL stability conditions. The general structure of the time-explicit coupling scheme is summarized in Fig. 6. The main difference with the scheme from [29] is the use of various solid particles (with possible fragmentation). The main difference with the scheme from [28] is the possibility of fragmentation while the latter employs a time semi-implicit approach to determine geometric quantities.

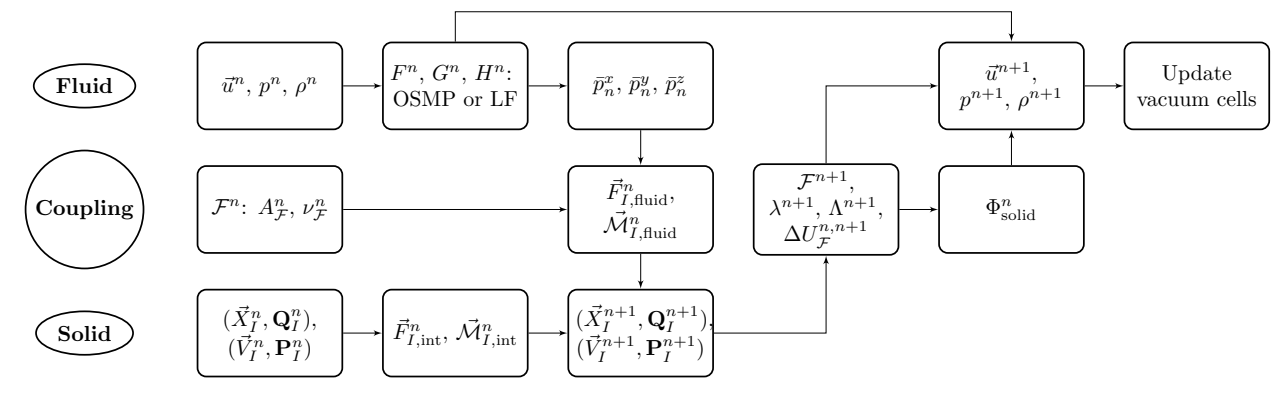

Figure 6: Time-explicit coupling scheme

\section{Numerical results}

In this section, we present numerical results. We first verify the conservation properties of the scheme and the propagation of the fluid in opening fractures in a three-dimensional structure. Then, we simulate the effect of an internal explosion in a steel cylinder in two space dimensions. Finally, we consider an overpressure inside a cube with mobile walls.

\subsection{Flow through opening fractures in $3 \mathrm{~d}$}

In this test case, we consider a three-dimensional structure composed of two particles and immersed in a fluid at rest. We assume that one particle is fixed, and that the other is displaced with a prescribed velocity, leading to an opening fracture between the two particles. As the opening velocity is high, a vacuum region is created between the two particles and is progressively filled with fluid. The fluid domain is the box $[0,2] \times[0,2] \times[0,2] \mathrm{m}$. The initial fluid state is given by $(\rho, \vec{u}, p)=\left(1 \cdot 4 \mathrm{~kg} \cdot \mathrm{m}^{-3}, \overrightarrow{0} \mathrm{~m} \cdot \mathrm{s}^{-1}, 1 \mathrm{~Pa}\right)$. The computation is carried out until $t=0.25 \mathrm{~s}$. Three uniform grids are considered for the fluid with reflecting boundary conditions. The first grid contains $(75 \times 75 \times 75)$ cells, the second $(100 \times 100 \times 100)$ cells, and the third grid $(125 \times 125 \times 125)$ cells. The solid is discretized with two parallelepipedic particles, having the same dimensions $(0.4,0.8,0.8) \mathrm{m}$ and their centers of mass are located at $(0.8,1,1) \mathrm{m}$ and $(1.2,1,1) \mathrm{m}$. The density of the solid is $\rho_{s}=7000 \mathrm{~kg} \cdot \mathrm{m}^{-3}$. The left particle is motionless, and the right particle has a prescribed velocity $\vec{V}=1 \vec{e}_{x} \mathrm{~m} \cdot \mathrm{s}^{-1}$.

Kinetic energy is transferred from the mobile particle to the fluid, and the particle displacement generates a compression wave in the fluid at the right of the moving particle, and a rarefaction wave propagates inside the opening fracture. In Fig. 7, we display 30 density iso-contours in the plane $\{z=1\} \mathrm{m}$ at times $0.07 \mathrm{~s}, 0.1 \mathrm{~s}, 0.15 \mathrm{~s}$, and $0.25 \mathrm{~s}$ using grid 3 . At time $t=0.07 \mathrm{~s}$, the gap is filled with fluid having low density compared to the density of the external fluid. At later times, the density between the two particles increases, and shock waves propagate over the fluid domain.

The pressure distribution along the line $\{x=1, z=1\} \mathrm{m}$ (this line passes through the middle of the opening fracture) on grids 1,2 , and 3 is shown in Fig. 8 at times $0.02 \mathrm{~s}$ and $0.12 \mathrm{~s}$. At time $t=0.02 \mathrm{~s}$ for the three grids the opening fracture begins to fill with fluid. Most of the fracture is still occupied by vacuum. The opening fracture is very small, and the fluid cells inside the fracture are small cut-cells. The pressure variations are related to the mixing procedure applied to these small cut-cells. The crack is progressively filled up with fluid. At time $t=0.12 \mathrm{~s}$, the crack has been totally filled with fluid. As the solid has continued to pull the fluid as a piston, the pressure is very low in the center of the opening fracture. The situation at the edges of the opening fracture resembles that of a shock tube: rarefaction waves are generated in the fluid near the opening edge, while compression waves in the fracture tend to 

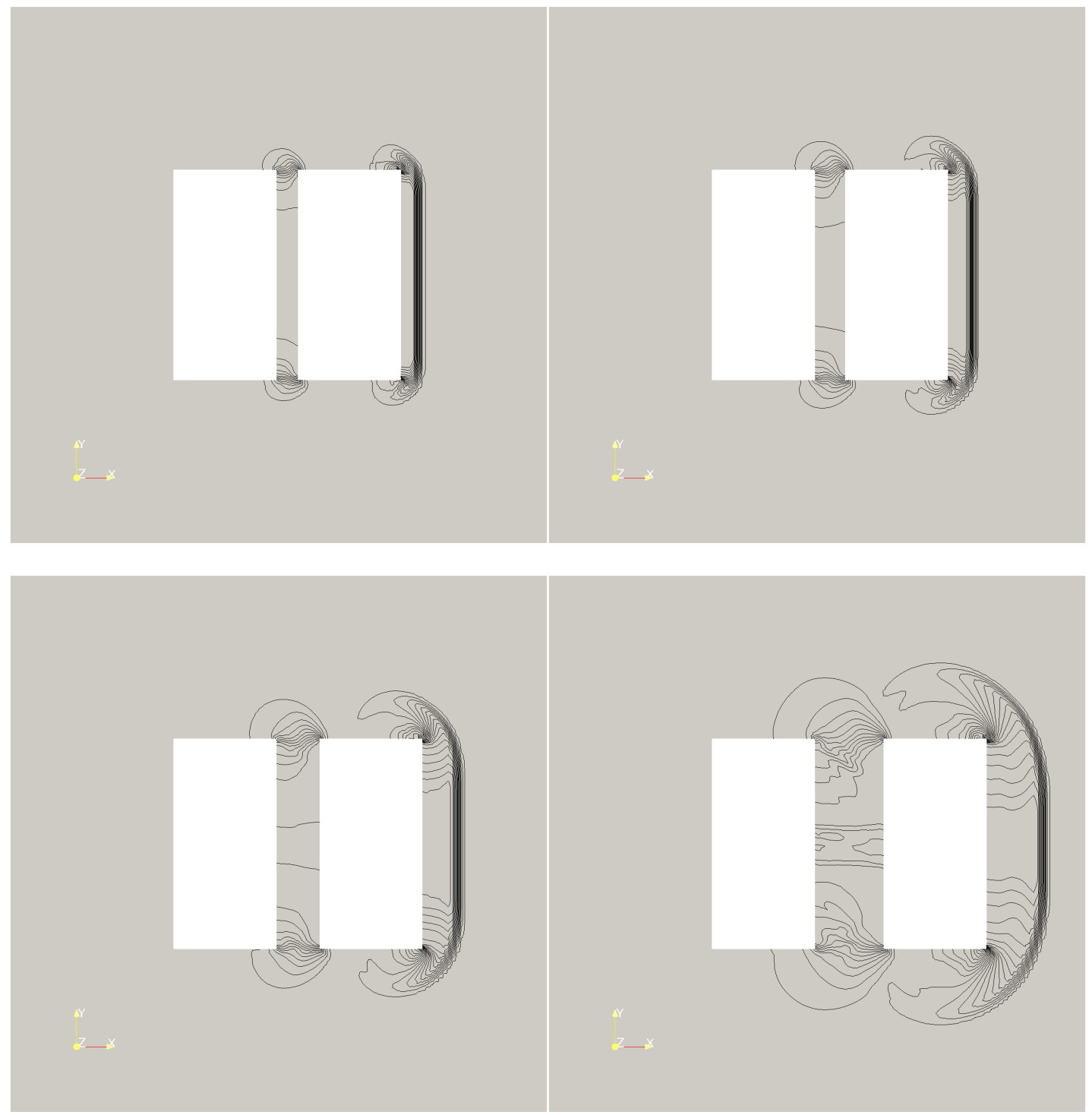

Figure 7: 30 density iso-contours in the plane $\{z=1\} \mathrm{m}$ on grid 3 at times $0.07 \mathrm{~s}, 0.1 \mathrm{~s}, 0.15 \mathrm{~s}$, and $0.25 \mathrm{~s}$ from left to right and top to bottom.
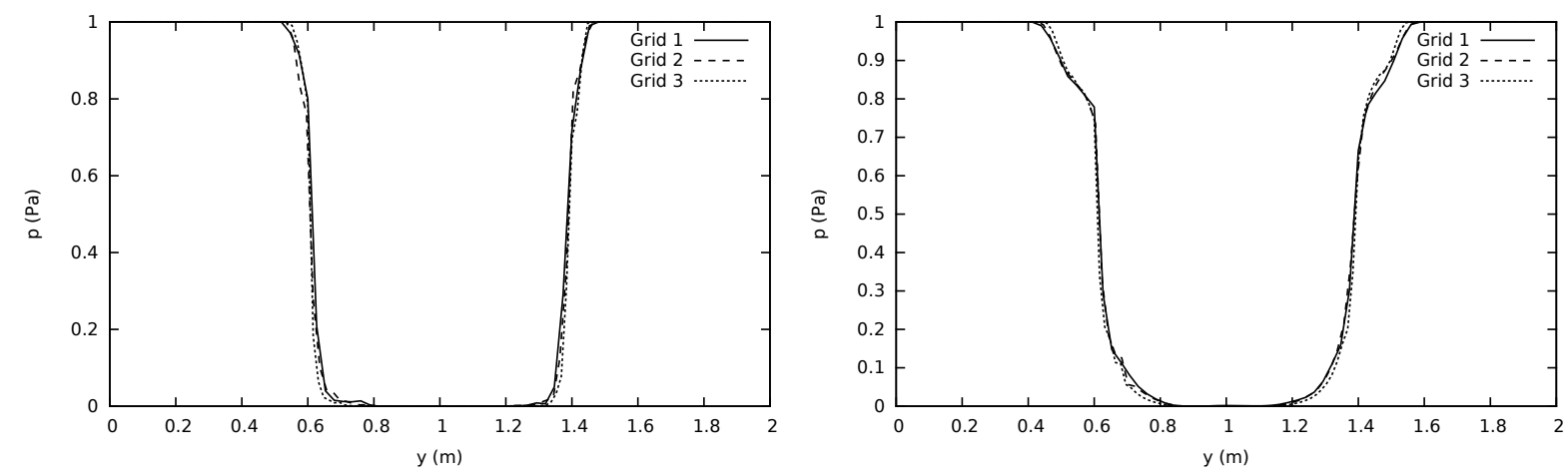

Figure 8: Pressure distribution along the line $\{x=1, z=1\} \mathrm{m}$ on grid 1 , grid 2 , and grid 3 at times $0.02 \mathrm{~s}$ (left) and $0.12 \mathrm{~s}$ (right). 
increase the pressure inside the fracture. We notice that the pressure inside the opening fracture is fairly well-converged, although the profiles remain slightly grid-dependent. The pressure difference is due to the fact that there is less numerical diffusion when the mesh is refined.

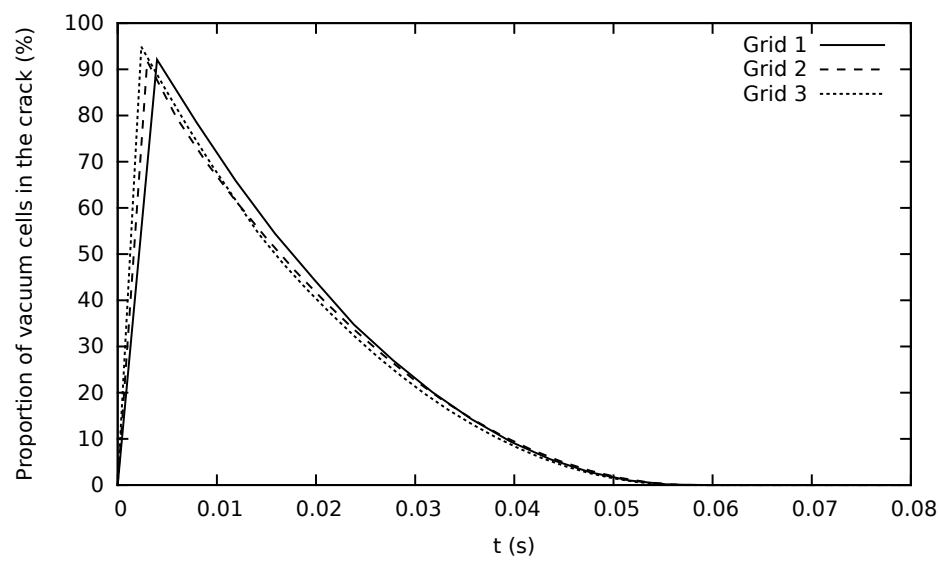

Figure 9: Time evolution of the proportion of vacuum cells on the crack interface for three fluid grids.

Fig. 9 presents the time evolution of the proportion of vacuum cells on the solid crack interface (in the plane $\{x=1\} \mathrm{m})$ for the three grids. We observe that, as expected, the proportion of vacuum cells reaches a peak immediately after the initial crack opening and then decreases to zero. We note that the proportion of vacuum cells reaches more than $90 \%$ as the whole crack is initially vacuum, except for the borders of the surface, and that vacuum cells disappear around $t=0.06 \mathrm{~s}$ for all three grids. Excellent convergence is achieved, suggesting that the presence of vacuum cells has physical relevance in the model considered here.

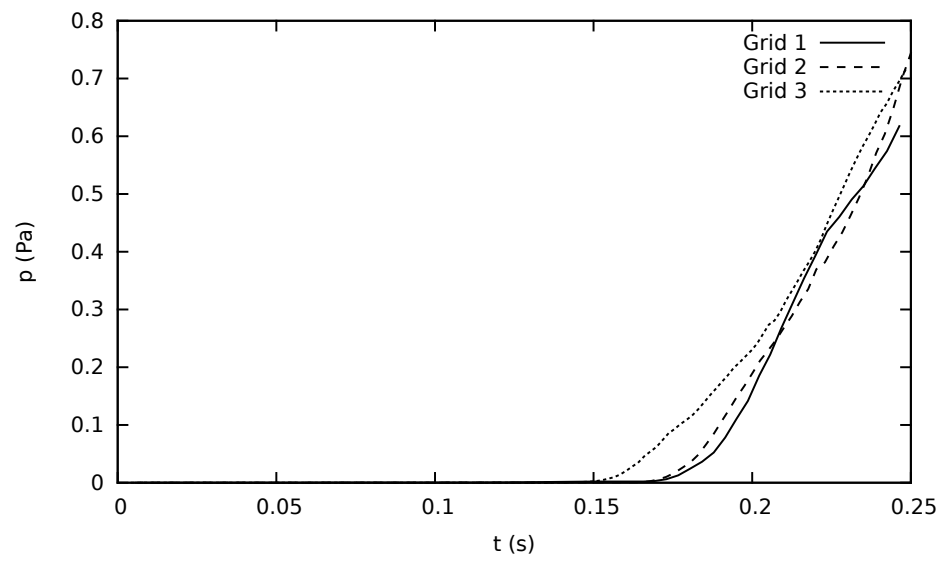

Figure 10: Time evolution of the pressure in the center of the opening fracture for three fluid grids.

Fig. 10 illustrates the time evolution of the pressure at the point $(1,1,1) \mathrm{m}$ situated at the center of the opening fracture for the three fluid grids. The center cell remains a vacuum cell until time $t=0.06 \mathrm{~s}$. It then becomes a fluid cell with a pressure close to zero due to the pulling apart of the solid block which leaves a state close to vacuum. The pressure increases once the compression waves from the surrounding fluid domain reach the center, tending to the outer pressure of $1 \mathrm{~Pa}$. We observe a difference in the time of arrival of the compression waves depending on grid refinement: around $t=0.17 \mathrm{~s}$ for grids 1 and 2 and around $t=0.15 \mathrm{~s}$ for grid 3 . We attribute this discrepancy to the fact that the celerity of waves is highly dependent on the pressure and density state inside the gap. The accuracy of the numerical flux scheme decreases significantly once the pressure and density jumps reach several orders of magnitude. Still, the general pattern of the pressure increase is preserved on all three grids.

In Fig. 11a we present the relative conservation error of fluid mass, computed as the difference between the initial mass and the mass at the different time steps for the three fluid grids. The mass difference 


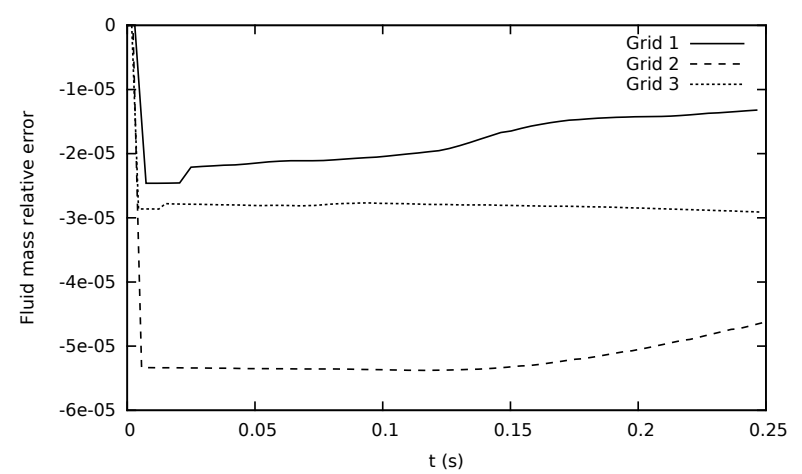

(a)

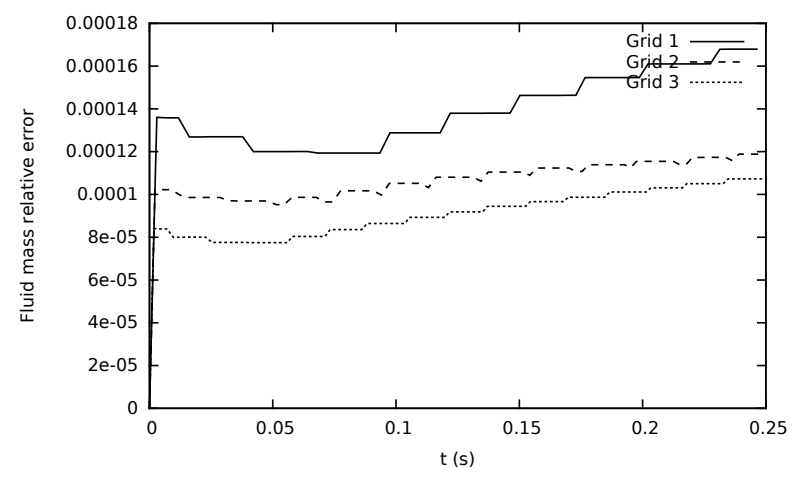

(b)

Figure 11: Relative conservation error on (a) fluid mass and (b) system energy for three fluid grids.

is normalized by the maximum amount of mass swept by the movement of the solid. In Fig. 11b we present the relative energy conservation error, computed as the difference between the initial energy and the energy at the different time steps for the three grids. This energy difference is normalized by the maximum energy exchange between the fluid and the solid. We observe that the relative conservation error on mass and energy is extremely low and decreases with grid refinement. In fact, these errors are at the same level as in the case of a rigid solid [29]. For instance, the relative mass error is as low as $0.003 \%$, and the relative energy error is as low as $0.01 \%$ for grid 3 . The main effect accounting for mass and energy variations are the round-off errors involved in the evaluation of geometric quantities in cut cells. Moreover, concerning energy, the time-integration scheme for the solid being symplectic, it preserves an approximate discrete energy over long-time simulations. This typically induces fluctuations of the exact discrete energy of the solid around a mean value [25]. Interactions between these fluctuations and the conservative fluid occur. However, the overall conservation of energy for the system is very good.

\subsection{Internal explosion in a steel cylinder in $2 \mathrm{~d}$}

In this test case, we simulate an internal explosion in a shell formed by a steel cylinder in two space dimensions. The cylinder is initially surrounded by gas at atmospheric pressure, and contains gas at the same pressure. An overpressure region is initiated inside the cylinder resulting in shock waves impinging the inner cylinder wall. The computational domain is the rectangle $[0,30] \times[0,15] \mathrm{m}$. The computation is performed on a $600 \times 300$ fluid grid. The boundaries of the domain are reflecting boundaries. The cylinder is centered at $(15,7.5) \mathrm{m}$ with a thickness of $0.2 \mathrm{~m}$ and an interior radius of $5 \mathrm{~m}$. The cylinder is discretized with 50, 100, and 200 particles along its circumference and 1 particle in thickness. Initially, the state of the gas is

$$
\begin{cases}\rho=99.935 \mathrm{~kg} \cdot \mathrm{m}^{-3}, \vec{u}=\overrightarrow{0} \mathrm{~m} \cdot \mathrm{s}^{-1}, p=50,662,500 \mathrm{~Pa} & \text { if }(x, y) \in D((13,7.5) \mathrm{m}, 1 \mathrm{~m}), \\ \rho=0.118 \mathrm{~kg} \cdot \mathrm{m}^{-3}, \vec{u}=\overrightarrow{0} \mathrm{~m} \cdot \mathrm{s}^{-1}, p=10,132.5 \mathrm{~Pa} & \text { otherwise }\end{cases}
$$

where $D\left(\left(x_{0}, y_{0}\right), R\right)$ denotes the disk centered at $\left(x_{0}, y_{0}\right)$ with radius $R$. In Fig. 12, we display the initial density field of the fluid and the initial position of the cylinder. The density and the Young modulus of the cylinder are, respectively, $\rho_{s}=7860 \mathrm{~kg} \cdot \mathrm{m}^{-3}$ and $E=210 \mathrm{GPa}$, with a Poisson ratio $\nu=0$. In this test case, the relative elongation at break is set to $1 \%$. The simulation time is $t=0.0244 \mathrm{~s}$.

After impacting the inner cylinder wall, the shock wave partially reflects, while part of its energy is transferred as kinetic energy to the cylinder. At the same time, the cylinder is deformed, and pressure waves travel along its surface. In Fig. 13, we show the density field in the fluid and the normal stress distribution in the cylinder at times $2.5 \mathrm{~ms}, 5 \mathrm{~ms}, 7 \mathrm{~ms}, 13 \mathrm{~ms}, 20 \mathrm{~ms}$, and $24.4 \mathrm{~ms}$ when the cylinder is discretized with 50 particles. In Fig. 14, we show the density field in the fluid and the normal stress distribution in the cylinder at time $24.4 \mathrm{~ms}$ when the cylinder is discretized with 100 and 200 particles. We notice that, in all three cases for the cylinder discretization, the rupture takes place at the left and right side of the cylinder, in approximately the same areas. Convergence of brittle fractures requires 


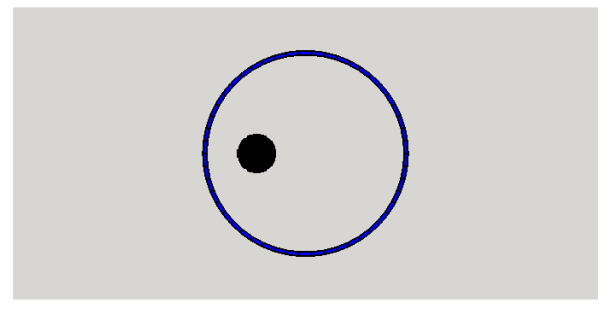

Figure 12: Density profile in the fluid and cylinder position at time $t=0 \mathrm{~s}$.
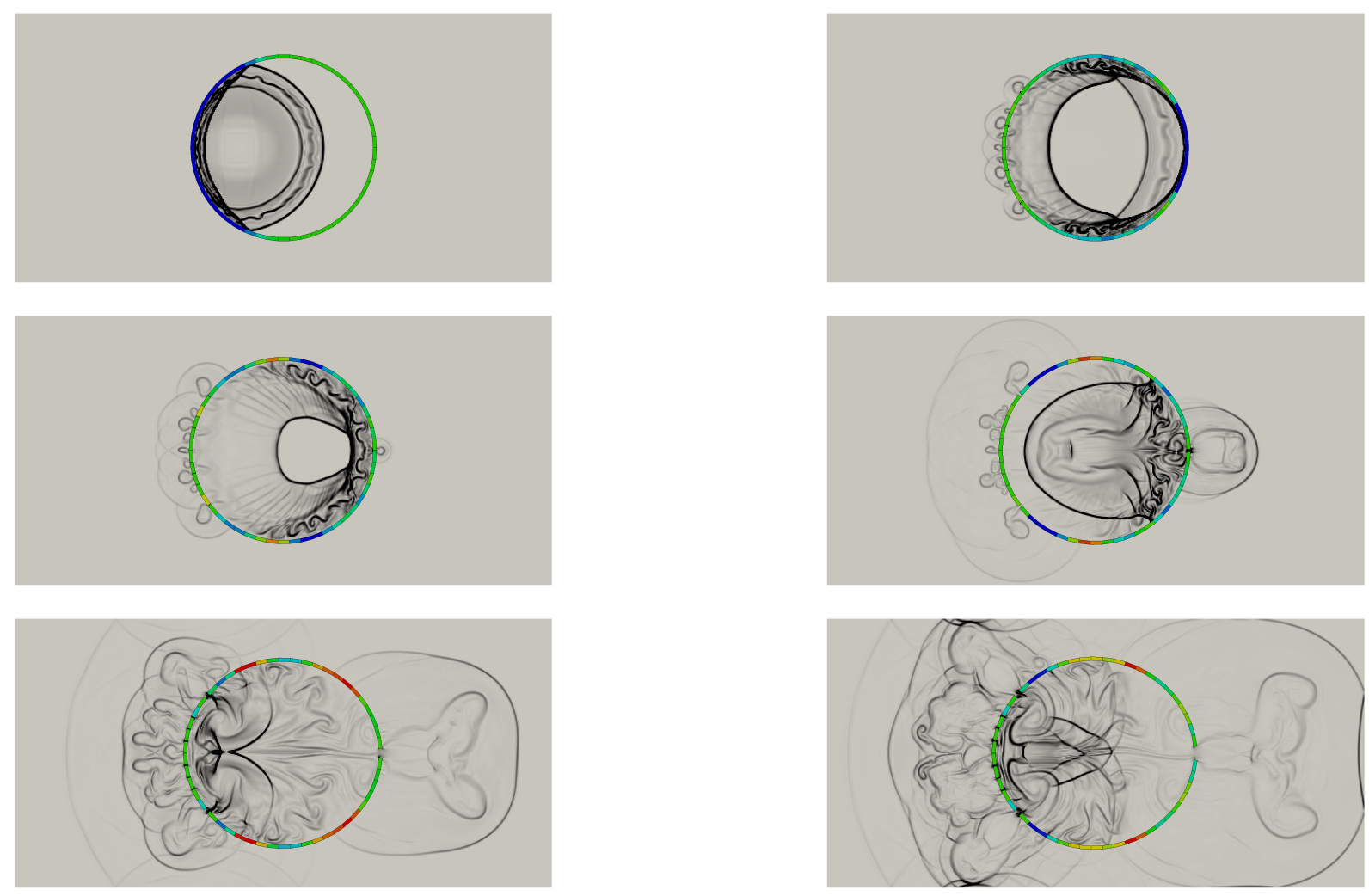

Figure 13: Density field in the fluid and normal stress distribution in the cylinder discretized with 50 particles at times $2.5 \mathrm{~ms}, 5 \mathrm{~ms}, 7 \mathrm{~ms}, 13 \mathrm{~ms}, 20 \mathrm{~ms}$, and $24.4 \mathrm{~ms}$ from left to right and from top to bottom.

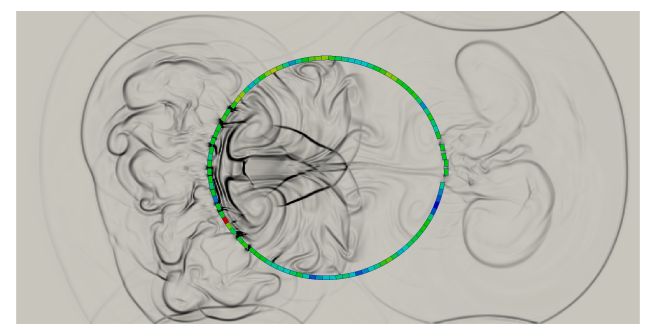

(a)

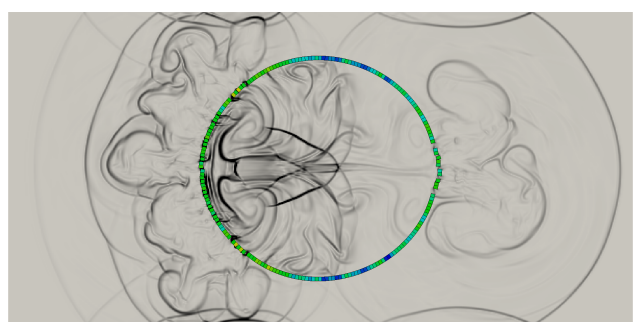

(b)

Figure 14: Density field in the fluid and normal stress distribution in the cylinder discretized with (a) 100 particles and (b) 200 particles at time $24.4 \mathrm{~ms}$. 
a large number of particles [23]. As the solid particles must have a thickness larger than or equal to two fluid grid cells, it would be necessary to use an adaptive meshing refinement in order to study the convergence of brittle fractures. We also notice that the fluid behavior is very similar inside the cylinder independently from the solid discretization. The main difference occurs in the regions where fluid flows through the cracks. The difference in crack patterns mainly changes the contact discontinuity pattern in the fluid outside the cylinder. The shock waves are reflected inside the cylinder and weak compression waves are transmitted by the movement of the solid outside the cylinder near the impacted regions of the cylinder. Several links between particles are broken, and the pressure waves in the solid propagate only in a few particles. We remark that the fluid penetrates into the opening gap between the particles and that the shock waves also propagate outside the cylinder. In Fig. 15, we illustrate the time evolution of the fluid pressure in the cell containing the point $(10,7.5) \mathrm{m}$ situated near the particle closest to the explosion inside the cylinder and in the cell containing the center of the domain $(15,7.5) \mathrm{m}$ for the three cylinder discretizations. We notice that the pressure patterns are remarkably similar independently from the solid discretization. In Fig. 15a, we notice that the pressure increases as the shock wave reaches the point, and then decreases as the shock wave is reflected by the solid boundary near this point. Peaks observed later on are due to reflected shock waves reaching the point after several other reflections on different points of the inner solid boundary. In Fig. 15b, the first peak corresponds to the shock wave passing through the point. The second one corresponds to reflected shock waves that reach the point after several reflections on the inner solid boundary. This second peak is stronger than the first one due to the refocusing of shock waves inside the cylinder.

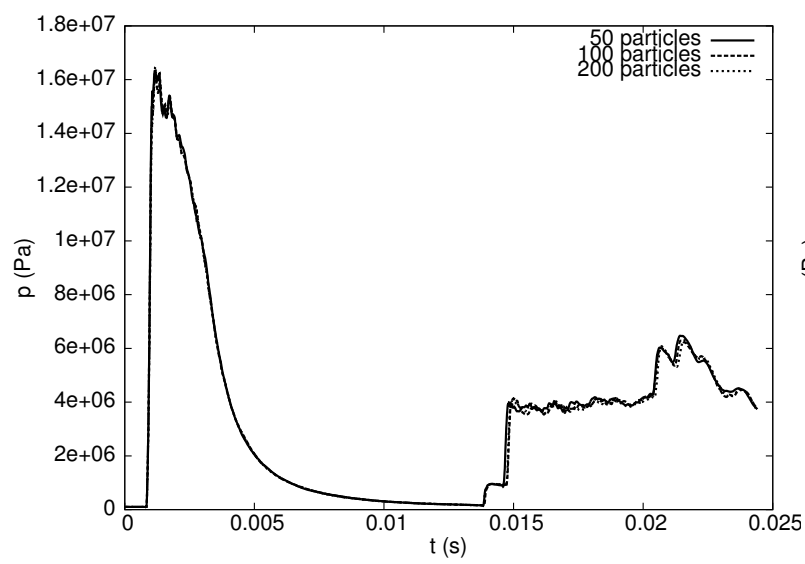

(a)

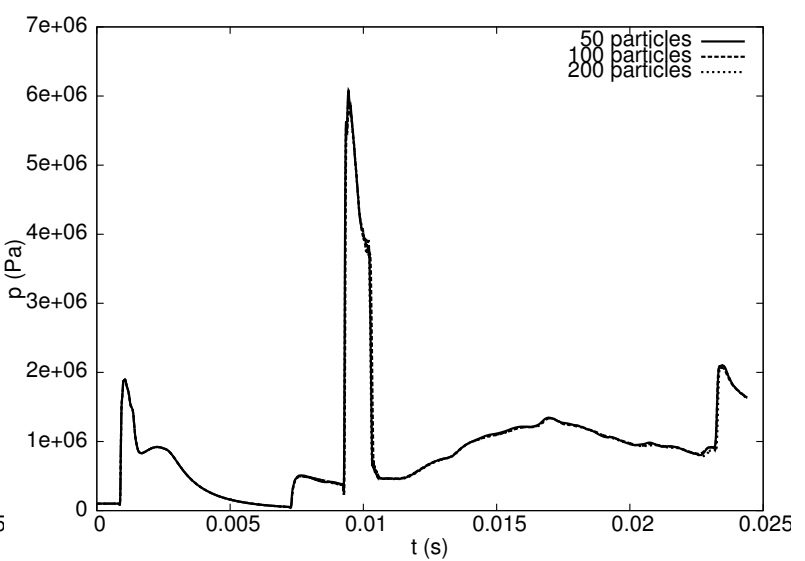

(b)

Figure 15: Time evolution of the fluid pressure in the cell containing (a) the point $(10,7.5) \mathrm{m}$ situated near the particle closest to the explosion inside the cylinder and (b) the point $(15,7.5) \mathrm{m}$ located at the center of the domain for the three cylinder discretizations.

Complex interaction between the waves travelling on the surface of the cylinder and the fluid occur, accounting for successive compression and traction phenomena in the cylinder leading to fractures located in the closest and farthest regions to the explosion. In Fig. 16, we display the time evolution of the normal stress in the solid particle closest to the explosion (centered initially at $(9.91006,7.82023) \mathrm{m}$ ) and in the solid particle farthest to the explosion (centered initially at $(20.0899,7.82023) \mathrm{m}$ ) when the cylinder is discretized with 50 particles. For the particle closest to the explosion, we notice that the normal stress decreases up to the time $t=2.6 \mathrm{~ms}$, where the link with one neighbor is broken. Immediately after this moment, the normal stress increases up to the time $t=3.2 \mathrm{~ms}$ when the link with the other neighbor is broken. As the particle has no link with other particles, the normal stress gets back to zero. For the particle farthest to the explosion, the normal stress decreases and reaches its minimum value at time $t=5 \mathrm{~ms}$, where the link with one neighbor is broken. After that, the normal stress oscillates with a smaller amplitude. The link with the other neighbor is not broken as the normal stress does not reach a high enough value. In Tab. 1, we report for each broken link the number of the two involved particles and the time when the link is broken when the cylinder is discretized with 50 particles. The particles are enumerated counter-clockwise, the first particle being centered initially at $(20.0899,7.82023) \mathrm{m}$. For the particles closer to the explosion, the links are broken when the shock 


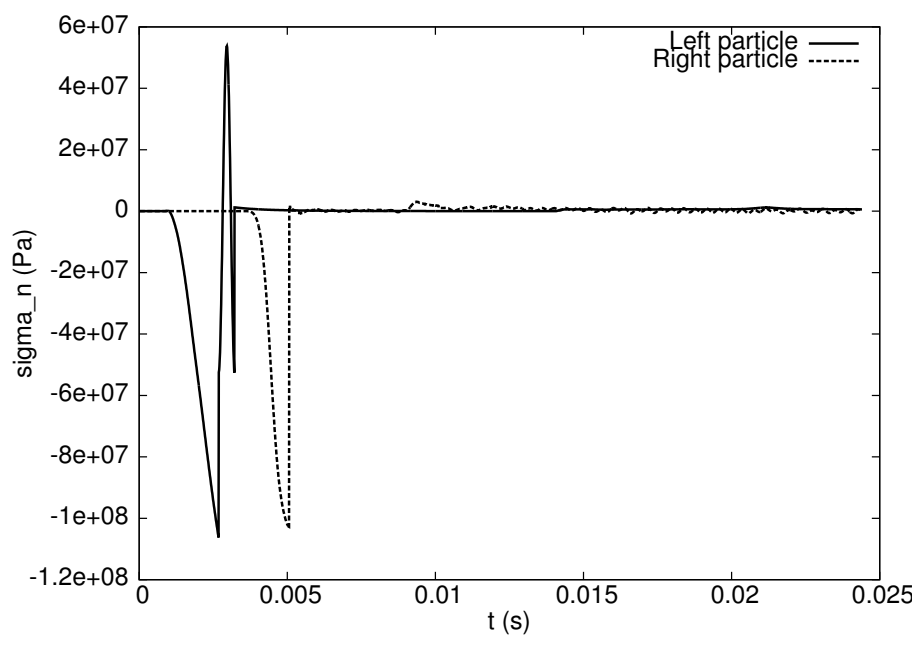

Figure 16: Evolution of the normal stress in the solid particle closest to the explosion (left particle) and in the solid particle farthest to the explosion (right particle) when the cylinder is discretized with 50 particles.

waves impact that region. For the particle farthest to the explosion, the links are broken when the waves travelling on the surface of the cylinder meet. In Fig. 17, we show the relative displacement between each

\begin{tabular}{|c|c|}
\hline Particles & Time $(\mathrm{ms})$ \\
\hline $23-24$ & 2.6 \\
$25-26$ & \\
$27-28$ & \\
\hline $20-21$ & 2.8 \\
$30-31$ & \\
\hline $24-25$ & 3.2 \\
$26-27$ & \\
\hline $22-23$ & 3.5 \\
$28-29$ & \\
\hline $1-50$ & 5 \\
\hline
\end{tabular}

Table 1: Broken links summary.

particle and its counter-clockwise neighbor at times $7 \mathrm{~ms}, 13 \mathrm{~ms}, 20 \mathrm{~ms}$, and $24.4 \mathrm{~ms}$ when the cylinder is discretized with 50 particles. We observe that the relative displacement between linked particles remains almost zero, whereas it is not the case for the particles with broken links. In Fig. 18, we show the relative displacement between each particle and its counter-clockwise neighbor at time $24.4 \mathrm{~ms}$ when the cylinder is discretized with 100 and 200 particles. The general patterns remain quite similar for the three cylinder discretizations: fragments are similarly located with more fragments on the left side close to the explosion compared to the right side. Incidentally, we observe that the symmetry is not exactly reproduced.

In Fig. 19, we display the displacement as a function of time of the center of mass of the solid particle closest to the explosion and that of the solid particle farthest to the explosion when the cylinder is discretized with 50 particles. We notice that the closest particle begins its movement along the $x$-axis around the time $t=2 \mathrm{~ms}$, while the farthest particle begins its movement along the same axis later on, at time $t=7 \mathrm{~ms}$. In both cases, the times correspond to a shock wave reaching the particle. The displacement of the closest particle has larger amplitude along the $x$-axis. The overpressure is higher near this particle, which broke the link with both of its neighbors, followed by a ballistic flight. Concerning the displacement along the $y$-axis, the closest particle exhibits very little displacement compared to the farthest particle. The farthest particle is still attached to other particles and is subjected to flexion moments from the rest of the solid.

In Fig. 20, we show the time evolution of the kinetic solid energy and the dissipated energy when the cylinder is discretized with 50 particles. The dissipated energy exhibits jumps whose amplitudes 

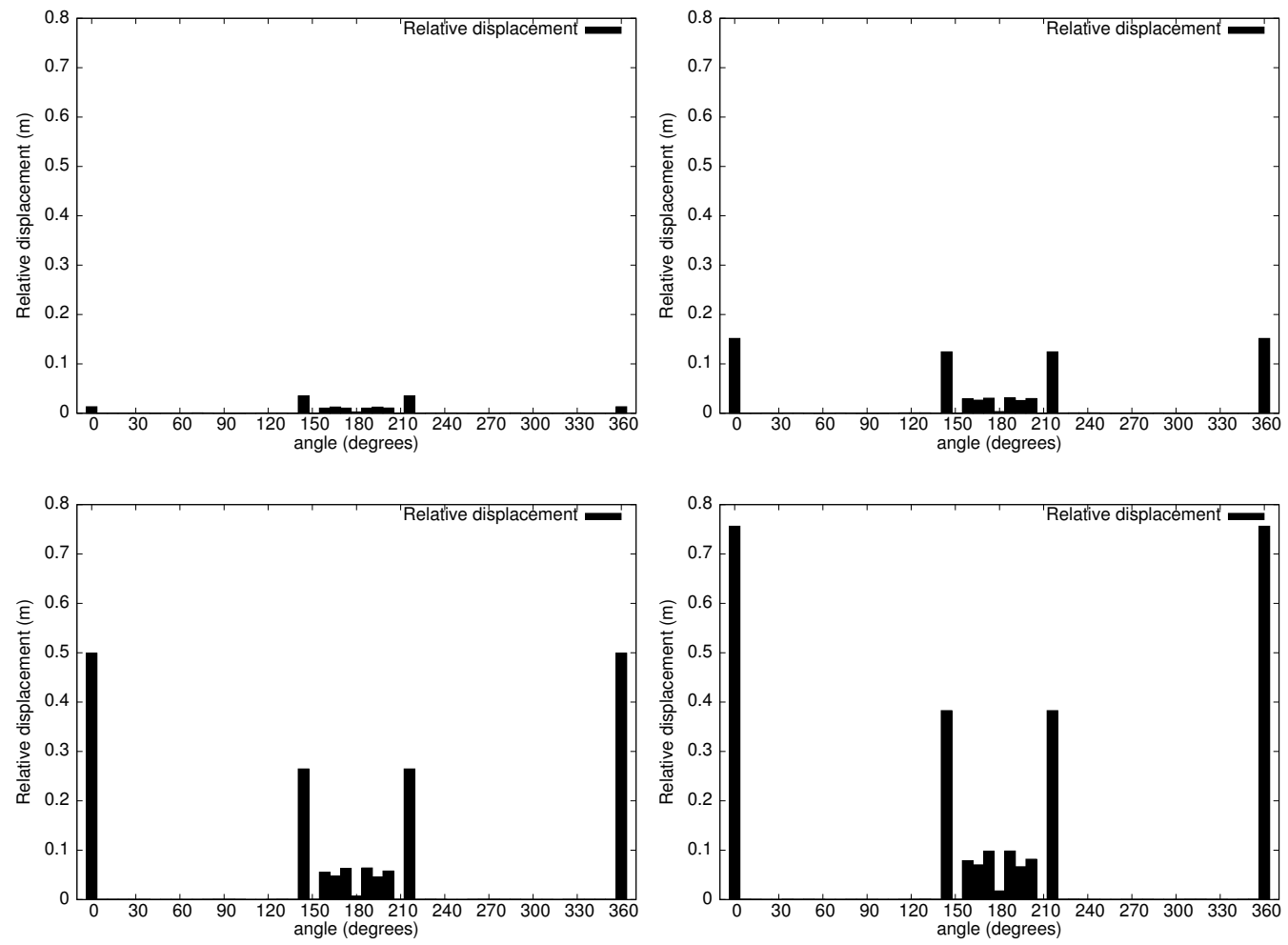

Figure 17: Relative displacement between each particle and its counter-clockwise neighbor at times $7 \mathrm{~ms}$, $13 \mathrm{~ms}$, $20 \mathrm{~ms}$, and $24.4 \mathrm{~ms}$ from left to right and from top to bottom when the cylinder is discretized with 50 particles.

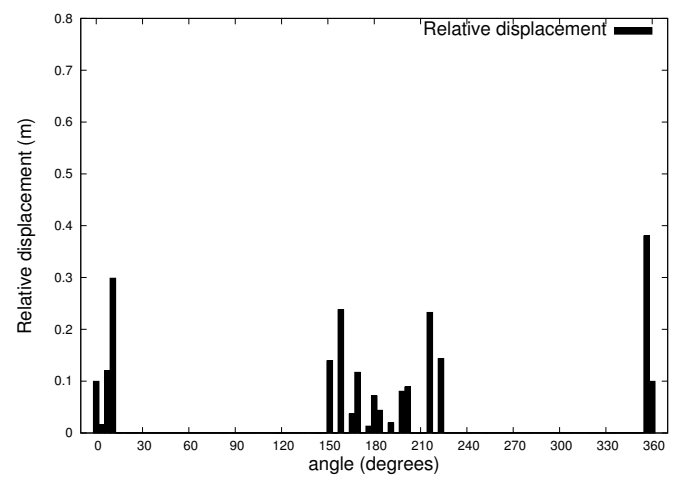

(a)

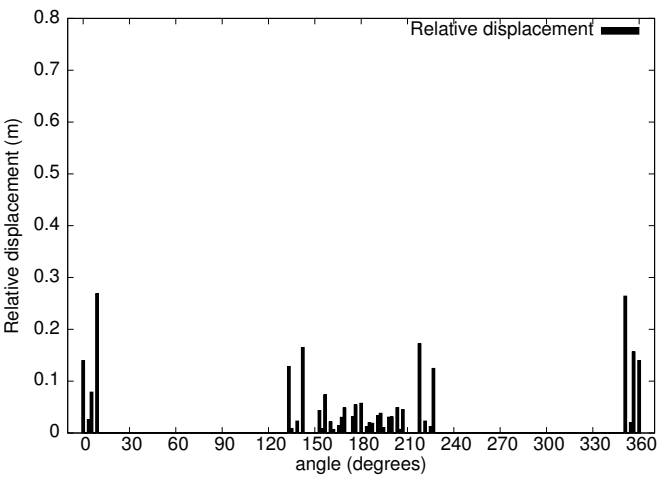

(b)

Figure 18: Relative displacement between each particle and its counter-clockwise neighbor at time $24.4 \mathrm{~ms}$ when the cylinder is discretized with (a) 100 particles and (b) 200 particles.

correspond to energy lost by breaking links between particles. In Fig. 21a, we present the relative conservation error of fluid mass (computed as before) for the three cylinder discretizations. As before, the main effect accounting for this variation are the round-off errors involved in the evaluation of geometric quantities in cut cells. The variation of mass is as low as $6 \cdot 10^{-7 \%}$ of the mass swept by the solid. In Fig. 21b, we present the relative energy conservation error, computed as the difference between the initial energy, the dissipated energy, and the energy at the different time steps for the three cylinder discretizations. This energy difference is normalized by the maximum energy exchange between the fluid and the solid. We observe a variation of both mass and energy. As before, the main effect accounting for this variation are the round-off errors involved in the evaluation of geometric quantities in cut cells 


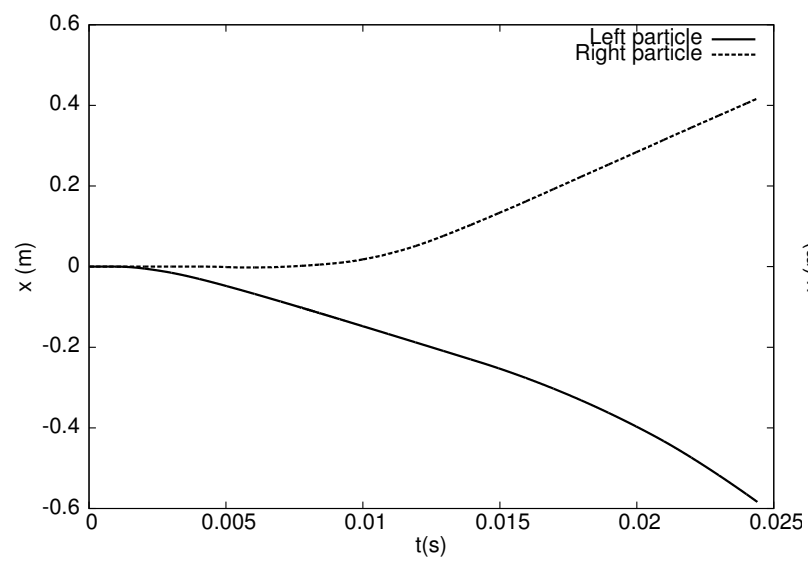

(a)

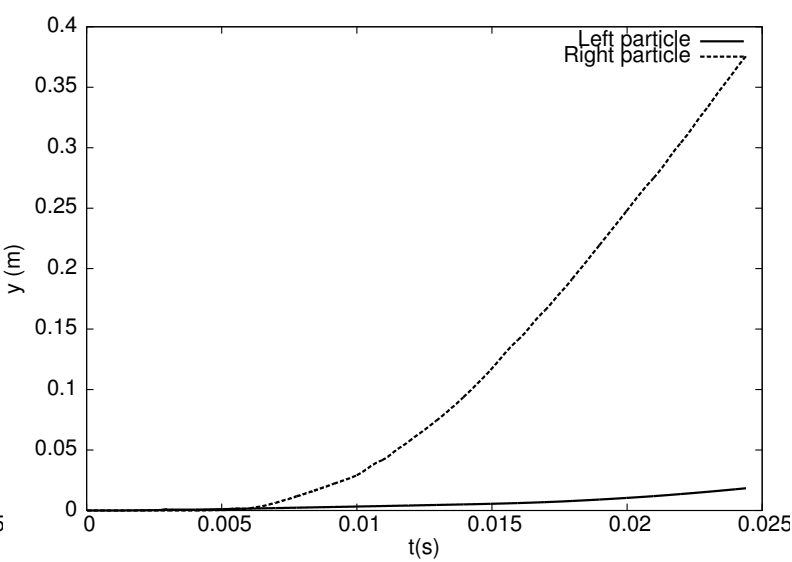

(b)

Figure 19: (a) $x$-coordinate and (b) $y$-coordinate of the center of mass of the solid particle closest to the explosion (left particle) and of the solid particle farthest to the explosion (right particle) as a function of time when the cylinder is discretized with 50 particles.

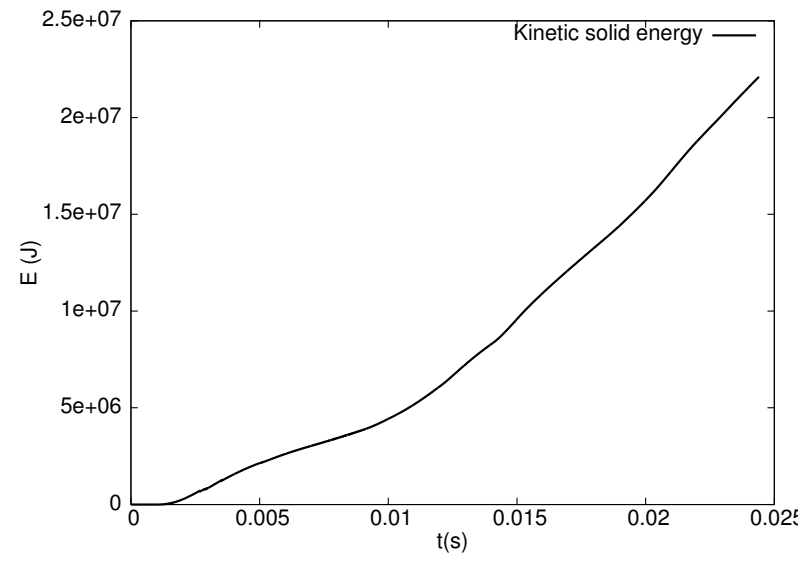

(a) Kinetic solid energy

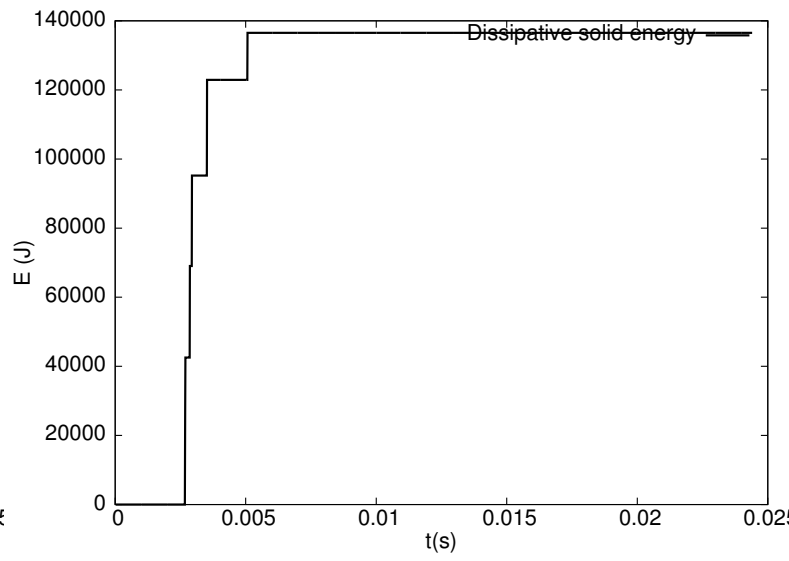

(b) Dissipated energy

Figure 20: Time evolution of the kinetic solid energy and of the dissipated energy when the cylinder is discretized with 50 particles.

and the symplectic time-integration scheme for the solid. When links break, extremely rapid changes in internal forces between particles occur. As the fracture does not occur in exactly the same way for the three discretizations, the energy changes accordingly. The variation of energy is as low as $0.1 \%$ of the energy exchange in the system in all three cases, which is a very low level of error.

\subsection{Overpressure inside a cube with mobile walls}

In this test case, an overpressure region is initiated inside a rigid cubic structure with mobile walls. In this test case, the rigid walls are not linked, but opening gaps appear between the solid walls as they are pushed away from each other by the shock wave produced by the overpressure. This test case allows us to verify the fluid penetration into opening gaps between solid particles driven by a fluid overpressure instead of having a prescribed translational velocity. The fluid domain is the box $[0,2] \times[0,2] \times[0,2] \mathrm{m}$, and the initial fluid state is given by

$$
\begin{cases}\rho=8 . \mathrm{kg} \cdot \mathrm{m}^{-3}, \vec{u}=\overrightarrow{0} \mathrm{~m} \cdot \mathrm{s}^{-1}, p=116.5 \mathrm{~Pa} & \text { if }(x, y) \in D((1.1,1 ., 1 .), 0.1) \mathrm{m}, \\ \rho=1.4 \mathrm{~kg} \cdot \mathrm{m}^{-3}, \vec{u}=\overrightarrow{0} \mathrm{~m} \cdot \mathrm{s}^{-1}, p=1 \mathrm{~Pa} & \text { otherwise }\end{cases}
$$




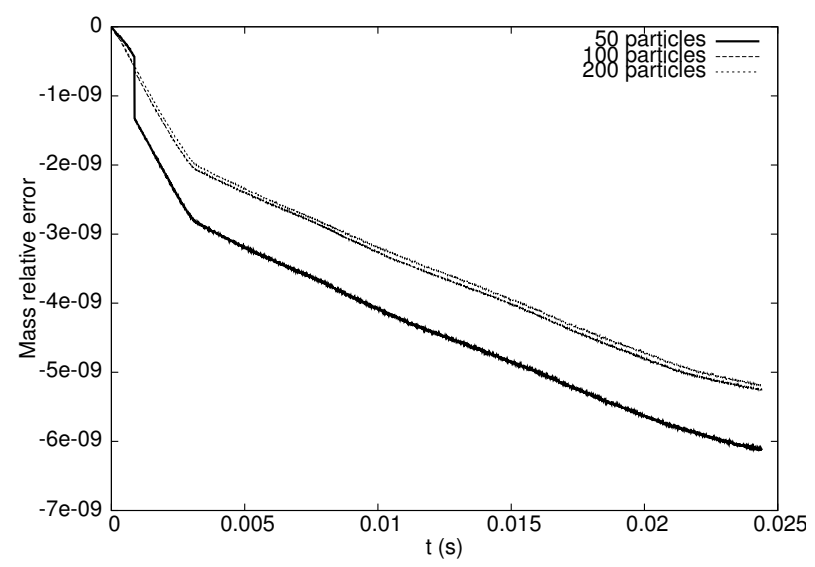

(a)

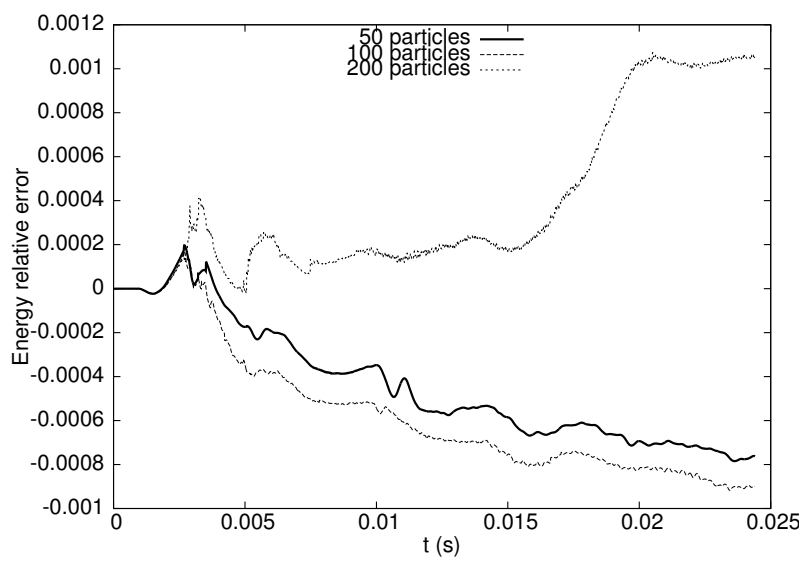

(b)

Figure 21: Relative conservation error on (a) fluid mass and (b) system energy for the three cylinder discretization.

where $D\left(\left(x_{0}, y_{0}, z_{0}\right), R\right)$ denotes the sphere centered at $\left(x_{0}, y_{0}, z_{0}\right)$ with radius $R$. The simulation time is $t=1 \mathrm{~s}$. The computation is performed on a $(100 \times 100 \times 100)$ fluid grid with periodic boundary conditions. The solid is composed of six particles. Each particle is a parallelepiped representing a mobile wall of the rigid cubic structure. The particles are described in Tab. 2. For each particle, we indicate the initial position of its center of mass (Center), and its dimensions $(\Delta x, \Delta y$, and $\Delta z)$.

\begin{tabular}{|l|c|c|c|c|}
\hline Particle & Center $(\mathrm{m})$ & $\Delta x$ & $\Delta y$ & $\Delta z$ \\
\hline 1 & $(0.65,1,1)$ & 0.1 & 0.8 & 0.6 \\
\hline 2 & $(1,0.65,1)$ & 0.6 & 0.1 & 0.6 \\
\hline 3 & $(1,1,0.65)$ & 0.8 & 0.8 & 0.1 \\
\hline 4 & $(1.35,1,1)$ & 0.1 & 0.8 & 0.6 \\
\hline 5 & $(1,1.35,1)$ & 0.6 & 0.1 & 0.6 \\
\hline 6 & $(1,1,1.35)$ & 0.8 & 0.8 & 0.1 \\
\hline
\end{tabular}

Table 2: Characteristics of the six particles forming the cubic structure.

The overpressure generates a shock wave impinging the solid. The shock wave propagates spherically and impacts first particle 4 as it is the closest to the overpressure zone. The shock wave is partially reflected, while part of its energy is transferred as kinetic energy to the particle. In Fig. 22, we display density iso-contours at times $0.07 \mathrm{~s}, 0.1 \mathrm{~s}, 0.14 \mathrm{~s}$, and $0.3 \mathrm{~s}$. The shock waves propagate inside the cubic structure, and reflect on particles leading to their displacements. We notice the displacement of particles due to energy transfer from the fluid. This leads to a gap between particles, so that the fluid can flow outside the cubic structure.

In Fig. 23, we display 30 density iso-contours in the plane $\{z=1\} \mathrm{m}$ at times $0.07 \mathrm{~s}, 0.1 \mathrm{~s}, 0.14 \mathrm{~s}$, and $0.3 \mathrm{~s}$. We notice that as soon as the displacement of the particles leads to gaps, the fluid leaks outside the cubic structure. We also observe a few weak compression waves in the exterior fluid due to the movement of the particles. In Fig. 24, we show the displacement as a function of time of the center of mass of each particle. For particles 1 and 4 , we display the $x$-coordinate of their center of mass because the displacements of these particles is along the $x$-axis due to the symmetry of the problem. Likewise, for particles 2 and 5, we display the $y$-coordinate of their center of mass, and for particles 3 and 6 , we display the $z$-coordinate of their center of mass. The overpressure is almost at the center of the solid, and the movement in the $x, y$, and $z$ directions are of same order of magnitude for the particles which have the same mass. The movement of the particles is smooth despite successive impinging shock waves. This is due to the effect of particles inertia. At the end of the simulation, the displacement of the particles is almost equal to half of the particles thickness.

The pressure distribution along the line $\{x=1.3, z=1\} \mathrm{m}$ is shown in Fig. 25 at times $0.07 \mathrm{~s}, 0.1 \mathrm{~s}$, $0.14 \mathrm{~s}, 0.3 \mathrm{~s}, 0.6 \mathrm{~s}$, and $0.9 \mathrm{~s}$. This line is close to the inner face of particle 4 . The pressure varies along the 

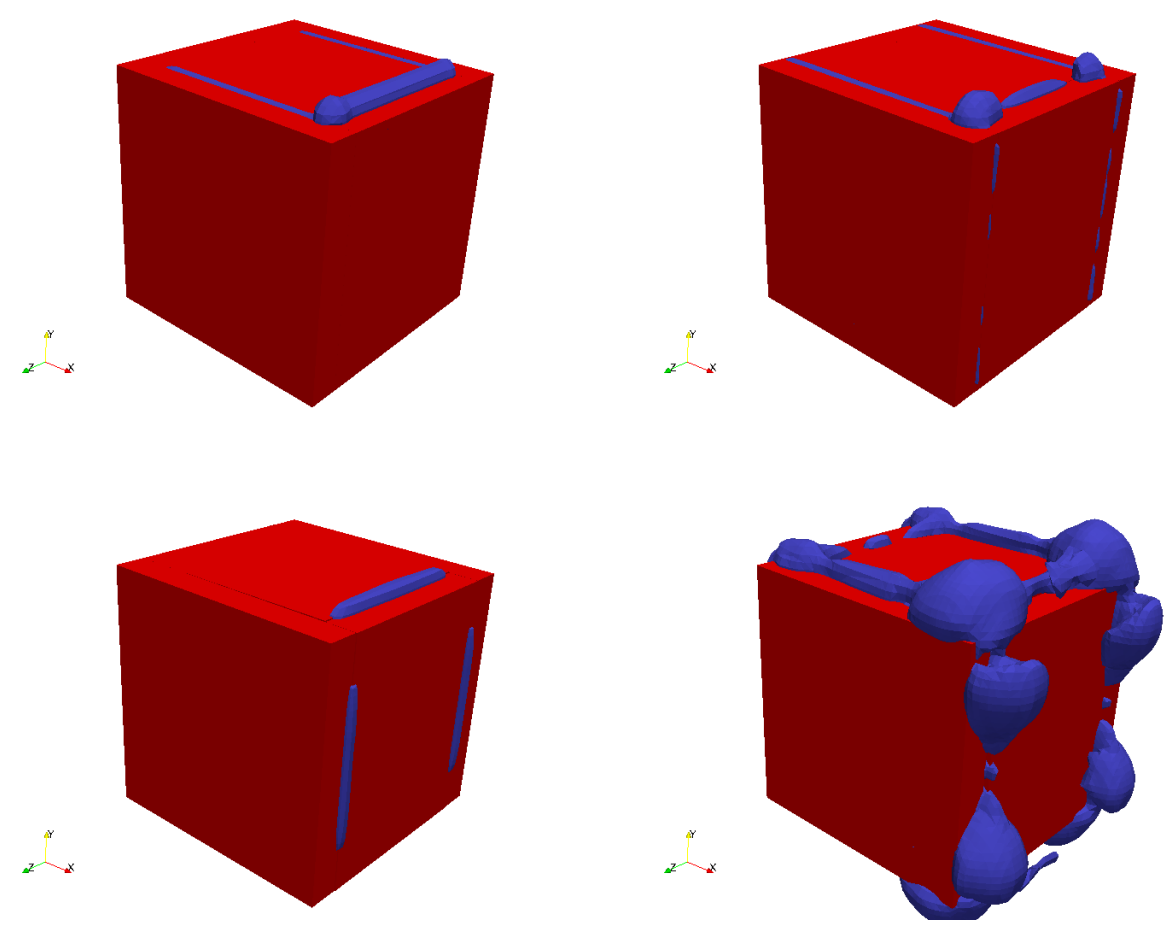

Figure 22: Density iso-contours at times $0.07 \mathrm{~s}, 0.1 \mathrm{~s}, 0.14 \mathrm{~s}$, and $0.3 \mathrm{~s}$ from left to right and from top to bottom.
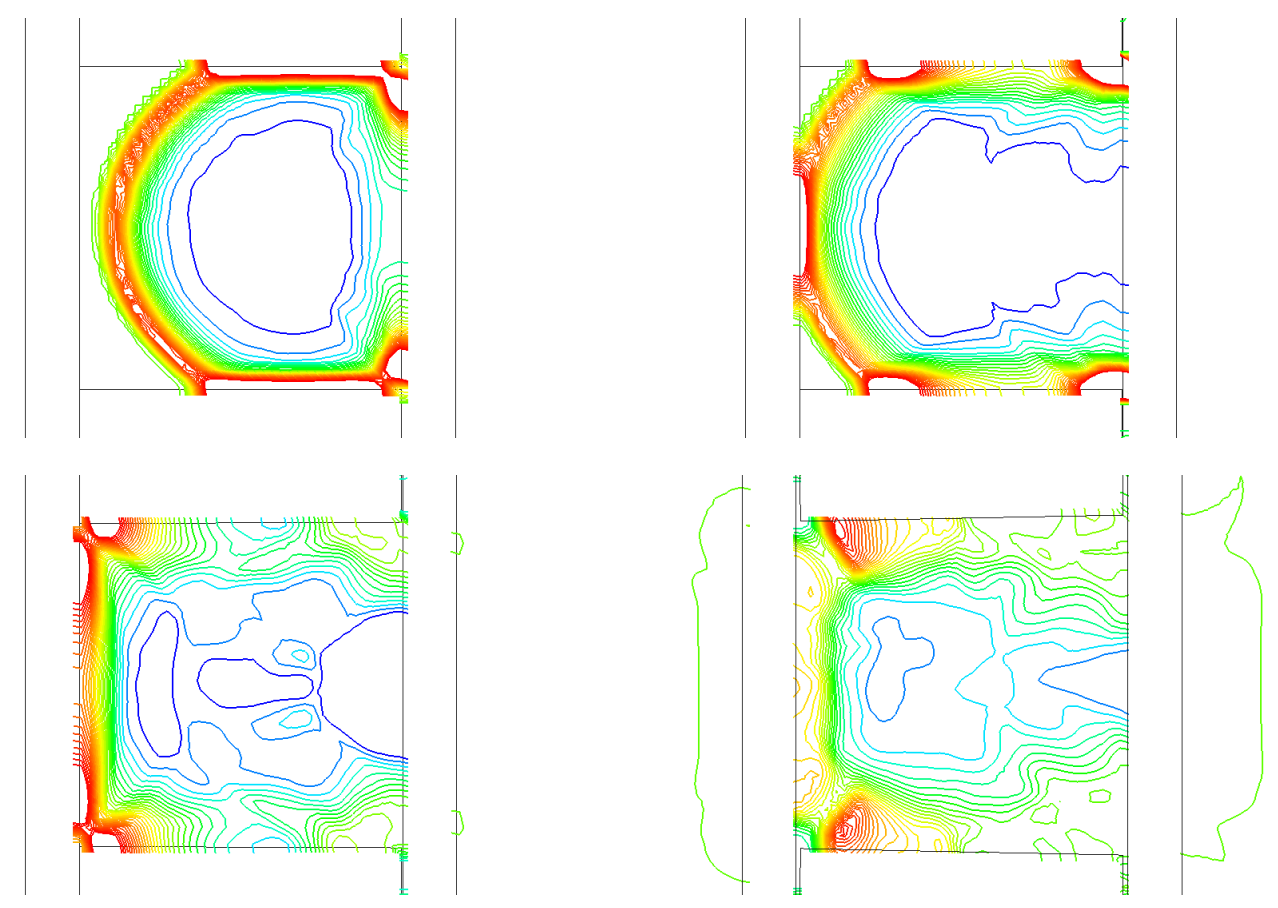

Figure 23: 30 density iso-contours in the plane $\{z=1\} \mathrm{m}$ at times $0.07 \mathrm{~s}, 0.1 \mathrm{~s}, 0.14 \mathrm{~s}$, and $0.3 \mathrm{~s}$ from left to right and from top to bottom.

$y$-axis. At time $t=0.07 \mathrm{~s}$, the pressure is high at both $y$-extremities of particle 4 . We observe in Fig. 24 that the displacement of particle 4 starts around this time. At time $t=0.1 \mathrm{~s}$ which corresponds to the 

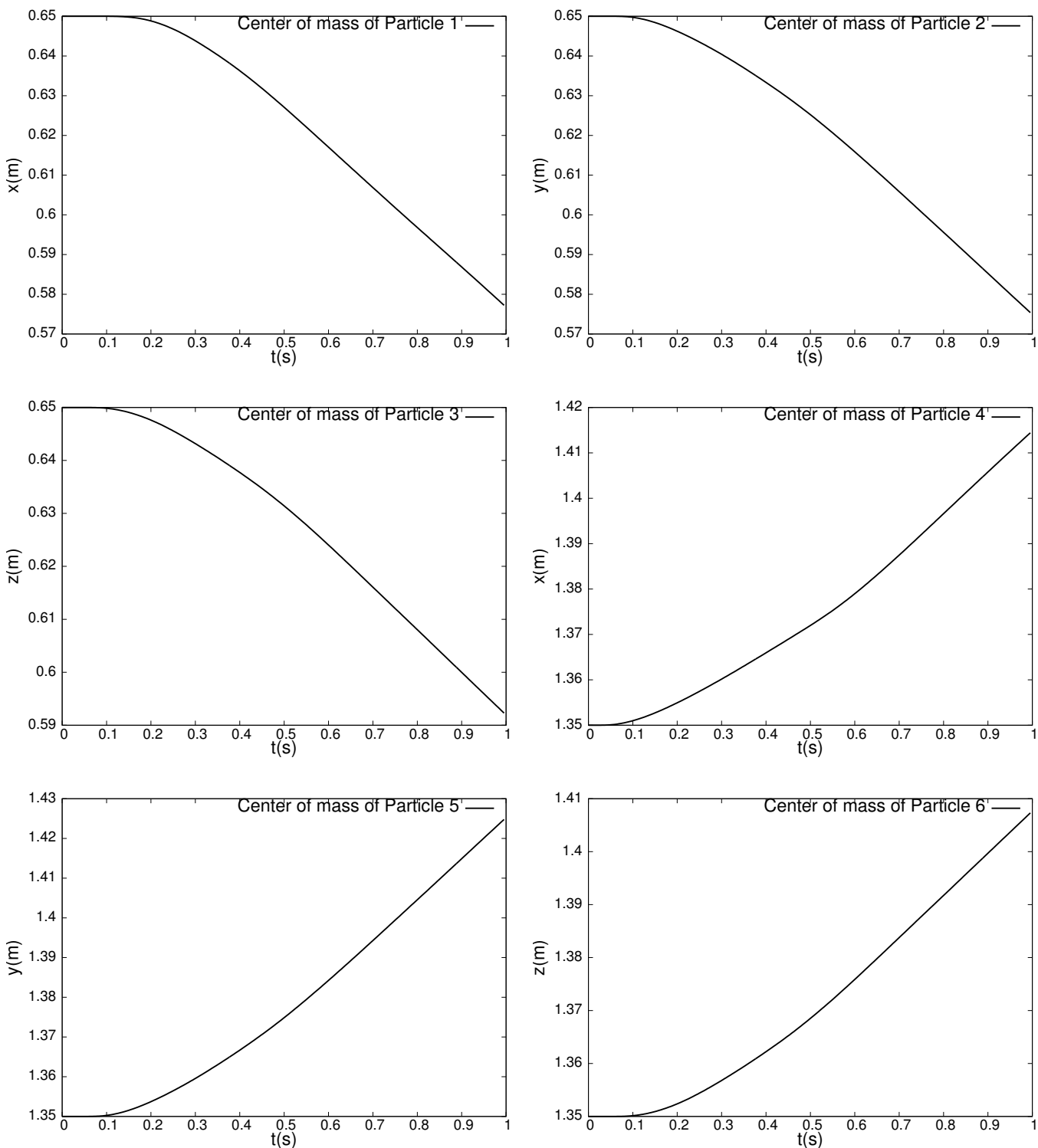

Figure 24: Position of the center of mass for the six particles.

beginning of the displacement of particles 3 and 6 , pressure has soared on the extremities while it remains steady in the middle of the face of the particle. At time $t=0.14 \mathrm{~s}$ which corresponds to the beginning of the displacement of particle 1, pressure has decreased even in the middle of the line (underpressure), and we notice a small compression wave corresponding to the leaking of the fluid through the gap. At time $t=0.3 \mathrm{~s}$, pressure has slightly increased along the line due to the arrival of the reflected waves on the solid face. The fluid continues to leak between the gaps, and at time $t=0.6$ we observe that the corresponding compression waves increase since the pressure near particle 4 decreases. At time $t=0.9 \mathrm{~s}$, the pressure is almost constant along the line.

Fig. 26a and Fig. 26b illustrate the time evolution of the pressure in the cell containing the points $(1.3,1.4,1) \mathrm{m}$ and $(1.3,0.6,1) \mathrm{m}$ respectively, which are situated at the outside corners of particle 4 . We observe initially a small decrease of pressure due to the fact that the fluid is sucked into the gap, then an increase of pressure due to the overpressure leaking from the cube through the opening between particles. The two curves maintain good symmetry despite the coarseness of the mesh. Fig. 26c illustrates the time evolution of the pressure in the cell containing the point $(1.3,1,1) \mathrm{m}$ which is located inside the cubic structure and near particle 4 . We observe the initial overpressure, and then the effect of the reflection of the shock waves on particles. 

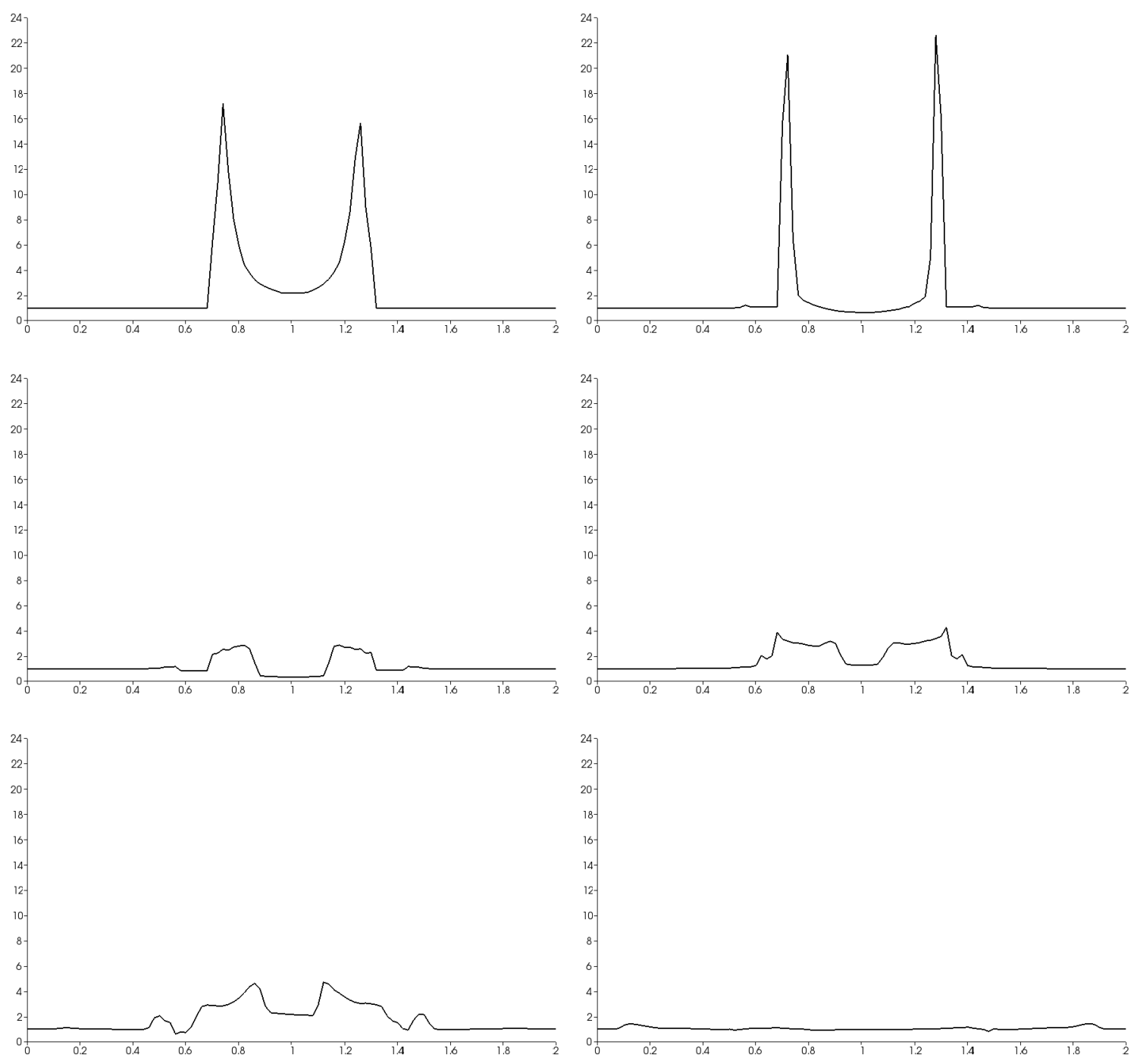

Figure 25: Pressure distribution along the line $\{x=1.3, z=1\} \mathrm{m}$ at times $0.07 \mathrm{~s}, 0.1 \mathrm{~s}, 0.14 \mathrm{~s}, 0.3 \mathrm{~s}, 0.6 \mathrm{~s}$, and $0.9 \mathrm{~s}$ from left to right and from top to bottom.

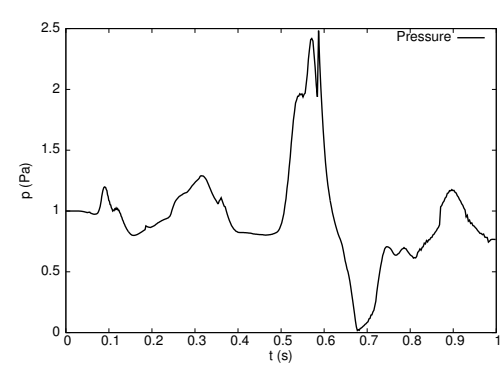

(a)

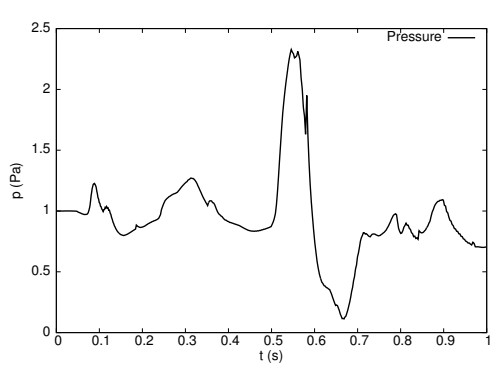

(b)

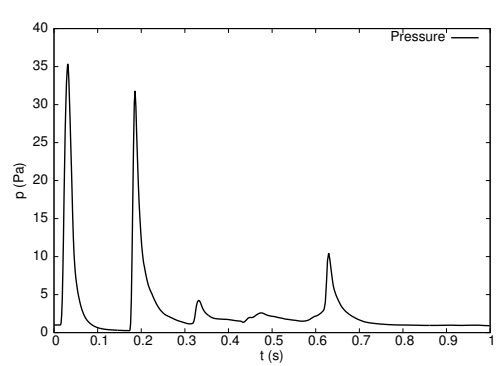

(c)

Figure 26: Evolution of the pressure in the cells containing (a) the point $(1.3,1.4,1) \mathrm{m}$, (b) the point $(1.3,0.6,1) \mathrm{m}$, and $(\mathrm{c})$ the point $(1.3,1,1) \mathrm{m}$.

Fig. 27 shows the evolution of the solid energy. Part of the shock waves energy is transferred to the particles as kinetic energy. This energy increases linearly to reach the value of $0.12 \mathrm{~J}$ at time $t=0.7 \mathrm{~s}$. In Fig. 28a, we present the relative conservation error of fluid mass (computed as before), and in Fig. 28b, we present the relative energy conservation error (computed as before). We observe a small variation of 


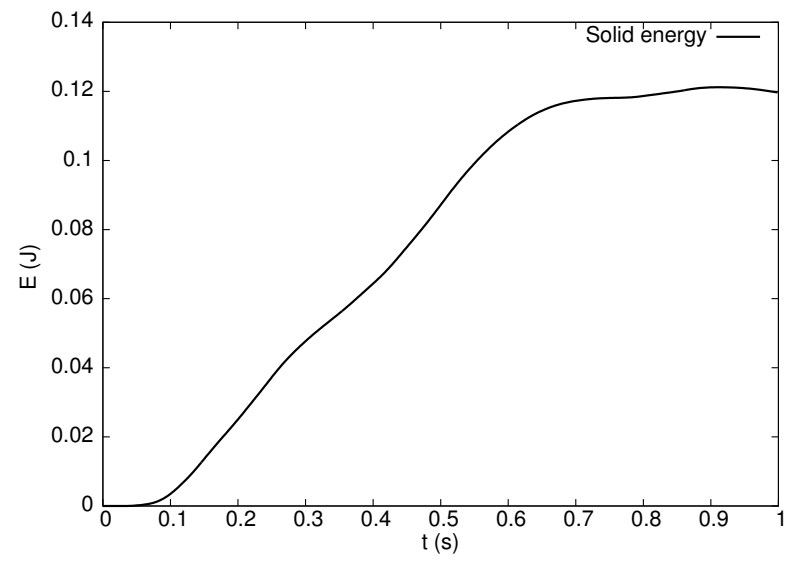

Figure 27: Time evolution of the solid energy.

both mass and energy. As before, the main effect accounting for this variation are the round-off errors involved in the evaluation of geometric quantities in cut cells and the symplectic time-integration scheme for the solid. The variation of mass is as low as $0.003 \%$ of the mass swept by the solid. The variation of energy is as low as $0.03 \%$ of the energy exchange in the system.

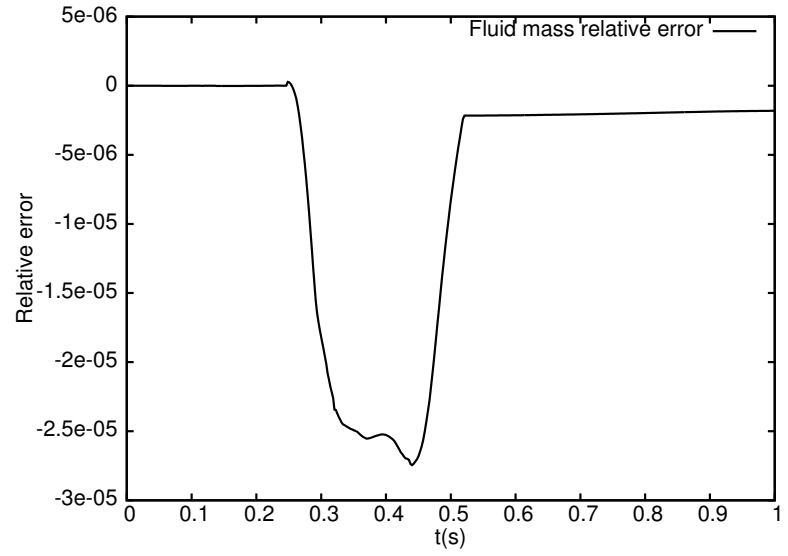

(a)

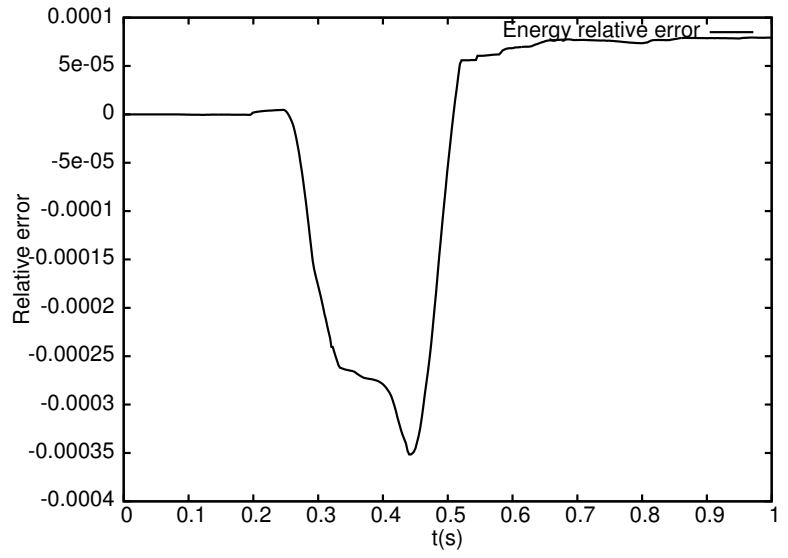

(b)

Figure 28: Relative conservation error on (a) fluid mass and (b) system energy.

\section{Conclusion}

In this work, we have developed a conservative method for the interaction between an inviscid compressible flow and a fragmenting structure. On the fluid side, we considered an inviscid Euler fluid in conservative form discretized by a Finite Volume method. On the solid side, we considered a fragmenting solid discretized by a Discrete Element method. An Embedded Boundary technique was employed through the modification of Finite Volume fluxes in the vicinity of the solid. During the process of fragmentation, vacuum between solid particles can occur, and the Lax-Friedrichs flux was employed to solve the corresponding Riemann problem. The coupling algorithm is based on an explicit time-marching procedure, it does not require remeshing of the fluid or solid domain, and allows fluid to pass through the fractured areas of the structure without any a priori knowledge of where fracture occurs.

The presented numerical simulations allowed us to illustrate the viability of the method in the case of two- and three-dimensional fragmenting solids coupled to an inviscid compressible flow with fluid flows through opening cracks. The prospect for continuing this work is to enrich the algorithm to take into account the possible contact between particles during the ballistic flight after fragmentation and to move 
on to more complex test cases. These developments are the subject of ongoing work.

Acknowledgement This work was supported in part by CEA/DAM.

\section{REFERENCES}

[1] G. T. Camacho and M. Ortiz. Computational modelling of impact damage in brittle materials. Int. J. Solids Structures, 33(20):2899-2938, 1996.

[2] F. Cirak, R. Deiterding, and S. P. Mauch. Large-scale fluid-structure interaction simulation of viscoplastic and fracturing thin-shells subjected to shocks and detonations. Computers $\&$ Structures, 85(11):1049-1065, 2007.

[3] P. Colella, D. T. Graves, B. J. Keen, and D. Modiano. A Cartesian grid embedded boundary method for hyperbolic conservation laws. J. Comput. Phys., 211(1):347-366, 2006.

[4] V. Daru and C. Tenaud. High order one-step monotonicity-preserving schemes for unsteady compressible flow calculations. J. Comput. Phys., 193(2):563-594, 2004.

[5] P. De Palma, M. D. De Tullio, G. Pascazio, and M. Napolitano. An immersed-boundary method for compressible viscous flows. Computers \& fluids, 35(7):693-702, 2006.

[6] R. Deiterding, F. Cirak, and S. P. Mauch. Efficient Fluid-Structure Interaction Simulation of Viscoplastic and Fracturing Thin-Shells Subjected to Underwater Shock Loading. International Workshop on Fluid-Structure Interaction, page 65, 2009.

[7] C. Denoual, G. Barbier, and F. Hild. A probabilistic approach for fragmentation of brittle materials under dynamic loading. Comptes Rendus de l'Académie des Sciences-Series IIB-Mechanics-PhysicsChemistry-Astronomy, 325(12):685-691, 1997.

[8] J. Dolbow, N. Moës, and T. Belytschko. An extended finite element method for modeling crack growth with frictional contact. Comput. Methods Appl. Mech. Eng., 190(51):6825-6846, 2001.

[9] J. Donea, S. Giuliani, and J. P. Halleux. An arbitrary Lagrangian-Eulerian finite element method for transient dynamic fluid-structure interactions. Comput. Methods Appl. Mech. Eng., 33(1):689-723, 1982.

[10] D. Doyen, A. Ern, and S. Piperno. Quasi-explicit time-integration schemes for dynamic fracture with set-valued cohesive zone models. Comput. Mech., 52(2):401-416, 2013.

[11] Z. Dragojlovic, F. Najmabadi, and M. Day. An embedded boundary method for viscous, conducting compressible flow. J. Comput. Phys., 216(1):37-51, 2006.

[12] E. A. Fadlun, R. Verzicco, P. Orlandi, and J. Mohd-Yusof. Combined immersed-boundary finitedifference methods for three-dimensional complex flow simulations. J. Comput. Phys., 161(1):35-60, 2000 .

[13] J. Falcovitz, G. Alfandary, and G. Hanoch. A two-dimensional conservation laws scheme for compressible flows with moving boundaries. J. Comput. Phys., 138(1):83-102, 1997.

[14] C. Farhat, A. Rallu, and S. Shankaran. A higher-order generalized ghost fluid method for the poor for the three-dimensional two-phase flow computation of underwater implosions. J. Comput. Phys., 227(16):7674-7700, 2008.

[15] R. P. Fedkiw. Coupling an Eulerian fluid calculation to a Lagrangian solid calculation with the ghost fluid method. J. Comput. Phys., 175(1):200-224, 2002.

[16] A. A. Griffith. VI. The Phenomena of Rupture and Flow in Solids. Phil. Trans. Roy. Soc.(Lon.) A, 221:163-198, 1920.

[17] D. Hartmann, M. Meinke, and W. Schröder. A strictly conservative Cartesian cut-cell method for compressible viscous flows on adaptive grids. Comput. Methods Appl. Mech. Eng., 200(9):1038-1052, 2011.

[18] X. Y Hu, B. C. Khoo, N. A. Adams, and F. L. Huang. A conservative interface method for compressible flows. J. Comput. Phys., 219(2):553-578, 2006.

[19] P. Le Tallec and J. Mouro. Fluid structure interaction with large structural displacements. Comput. Methods Appl. Mech. Eng., 190(24):3039-3067, 2001.

[20] P. D. Lea. Fluid Structure Interaction with Applications in Structural Failure. PhD thesis, Northwestern University, 2013.

[21] R. J. LeVeque. Finite volume methods for hyperbolic problems, volume 31. Cambridge university press, 2002. 
[22] C. Mariotti and L. Monasse. From general mechanics to discontinuity, unified approach to elasticity. Presses des Ponts, 2012.

[23] V. Michaut. Modeling of the dynamic fragmentation using a discrete element method/ Modélisation de la fragmentation dynamique par la méthode des éléments discrets. PhD thesis, Ecole Centrale Paris, France, 2011.

[24] L. Monasse, V. Daru, C. Mariotti, S. Piperno, and C. Tenaud. A conservative coupling algorithm between a compressible flow and a rigid body using an Embedded Boundary method. J. Comput. Phys., 231(7):2977-2994, 2012.

[25] L. Monasse and C. Mariotti. An energy-preserving Discrete Element Method for elastodynamics. ESAIM, Math. Model. Numer. Anal., 46:1527-1553, 2012.

[26] R. B. Pember, J. B. Bell, P. Colella, W. Y. Crutchfield, and M. L. Welcome. An adaptive Cartesian grid method for unsteady compressible flow in irregular regions. J. Comput. Phys., 120(2):278-304, 1995.

[27] C. S. Peskin. Numerical analysis of blood flow in the heart. J. Comput. Phys., 25(3):220-252, 1977.

[28] M. A. Puscas, V. Daru, A. Ern, C. Mariotti, L. Monasse, and C. Tenaud. Conservative coupling method between an inviscid compressible flow and a deformable structure. Submitted, http://hal.archives-ouvertes.fr/hal-00993324, 2014.

[29] M. A. Puscas and L. Monasse. A three-dimensional conservative coupling method between an inviscid compressible flow and a moving rigid solid. Submitted, http://hal.archives-ouvertes.fr/hal-00974602, 2014.

[30] T. Rabczuk, R. Gracie, J.-H. Song, and T. Belytschko. Immersed particle method for fluid-structure interaction. Int. J. Numer. Methods Engineering, 81(1):48-71, 2010.

[31] P. Schwartz, M. Barad, P. Colella, and T. Ligocki. A Cartesian grid embedded boundary method for the heat equation and Poisson's equation in three dimensions. J. Comput. Phys., 211(2):531-550, 2006.

[32] G. Strang. On the construction and comparison of difference schemes. SIAM Journal Numer. Anal., $5(3): 506-517,1968$.

[33] N. Sukumar and T. Belytschko. Arbitrary branched and intersecting cracks with the extended finite element method. Int. J. Numer. Meth. Eng., 48:1741-1760, 2000.

[34] J. W. Swegle and S. W. Attaway. On the feasibility of using smoothed particle hydrodynamics for underwater explosion calculations. Comput. Mech., 17(3):151-168, 1995.

[35] E. F. Toro. Riemann solvers and numerical methods for fluid dynamics, volume 16. Springer, 1999.

[36] Y. H. Tseng and J. H. Ferziger. A ghost-cell immersed boundary method for flow in complex geometry. J. Comput. Phys., 192(2):593-623, 2003. 